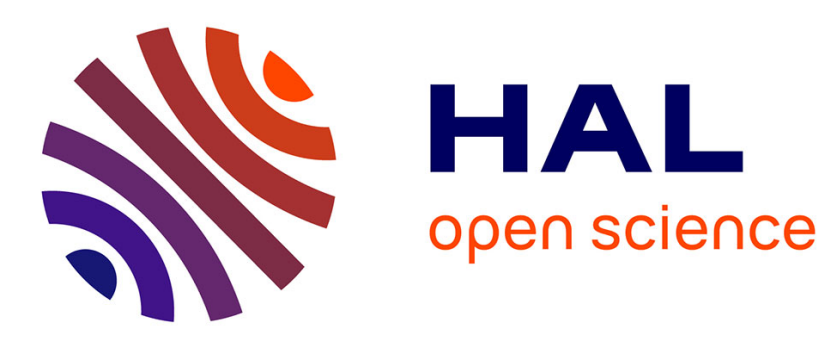

\title{
FFT-based modelling of transformation plasticity in polycrystalline materials during diffusive phase transformation
}

\author{
Takayuki Otsuka, Renald Brenner, Brigitte Bacroix
}

\section{To cite this version:}

Takayuki Otsuka, Renald Brenner, Brigitte Bacroix. FFT-based modelling of transformation plasticity in polycrystalline materials during diffusive phase transformation. International Journal of Engineering Science, 2018, 127, pp.92-113. 10.1016/j.ijengsci.2018.02.008 . hal-01826587

\section{HAL Id: hal-01826587 \\ https://hal.sorbonne-universite.fr/hal-01826587}

Submitted on 29 Jun 2018

HAL is a multi-disciplinary open access archive for the deposit and dissemination of scientific research documents, whether they are published or not. The documents may come from teaching and research institutions in France or abroad, or from public or private research centers.
L'archive ouverte pluridisciplinaire HAL, est destinée au dépôt et à la diffusion de documents scientifiques de niveau recherche, publiés ou non, émanant des établissements d'enseignement et de recherche français ou étrangers, des laboratoires publics ou privés. 


\title{
FFT-based modelling of transformation plasticity in polycrystalline materials during diffusive phase transformation
}

key words; Transformation Plasticity, Steel, FFT, Crystal Plasticity

\begin{abstract}
During heat treatment processes of steel products, transformation plasticity is known to play an important role as it affects the final product quality such as shape and residual stresses. To investigate this well-known phenomenon, a model coupling crystal plasticity with diffusive phase transformation is developed by using a fast Fourier transform (FFT) numerical scheme. Diffusional transformation including a plastically accommodated volume change is considered (i.e the Greenwood-Johnson mechanism). The model is then used to determine the pre-hardening effect on transformation plasticity. It is revealed that pre-hardening results in an anisotropic transformation strain; pre-tension decreases transformation strain and pre-compression increases transformation expansion along the pre-hardened direction. The model is also used to assess the existing analytical model developed by Leblond and Taleb. In this attempt, some features that contribute to the transformation plasticity are discussed. Among those, it is found that plastic deformation in daughter phase is non-negligibly small especially in the end of phase transformation. These analytical solutions predict linear relation between applied stress and transformation plastic strain. This reasoning attributes the linear relation to the solution of equivalent plastic strain increase with transformation by only the transformation expansion effect neglecting the applied stress effect.
\end{abstract}

\section{Introduction}

During the thermomechanical treatments involved in steel processing, transformation plasticity or transformation induced plasticity (TRIP) occurs as a result of the variation of the volumic fraction of the phases during the solid state transformation. Two main mechanisms are classically invoked: (i) a displacive mechanism (i.e. Magee effect [1]) with a shape change during transformation and (ii) a diffusive mechanism (i.e. Greenwood-Johnson effect [2]), implying nucleation and growth steps, with a volume change plastically accommodated [3]. They both have been the subject of a number of experimental studies (see, among others [4][5][6][7][8][9][10]).

The phenomenon is known to play a central role during phase transformation in defining the final shape and residual stress state of heat treated materials. Phenomenological models have been developed and been used in the framework of finite element thermo-mechanical simulations [11][12][13].

The micromechanical modelling of transformation plasticity dates back to the pioneering theoretical 
work of Leblond et al. [14] based on a rigorous homogenisation procedure. Few years later, an approximate analytical model has been derived from [15][16] to describe transformation plasticity due to the Greenwood-Johnson mechanism. Since then, developments have been suggested based on this approach [17]. Besides, it is worth mentioning that a variety of mean-field models have been developed; see, for instance, [18][19][20]. Apart from these works, numerical micromechanical modelling has also been performed, making use of the finite-element method (FEM) to study diffusional transformations. With increasing complexity, numerical investigations have first considered the case of a two-phase material with $J_{2}$ plasticity, and various nucleation rules, $[14][15][21][22][23]$, and more recently, the case of polycrystalline materials with crystalline plasticity at the slip system level, together with a microstructure described by a Poisson-Voronoi tessellation [24].

In the context of classical plasticity (i.e. without solid phase transformation), an efficient numerical scheme based on fast Fourier transform (FFT) [25] has been successfully applied to a variety of problems and constitutive relations [26][27][28][29][30]. This alternative approach to FEM allows to consider large polycrystalline aggregates with reasonable CPU time and memory allocation. Its accuracy has been discussed by confronting with FEM simulation results [31]. Besides, meshing of the microstructure is not necessary as the computation is directly made on the digital image of the material (regular grid of pixels in $2 \mathrm{D}$ or voxels in $3 \mathrm{D}$ ). These features are especially convenient to consider experimental microstructural data obtained by fine-scale EBSD or X-ray diffraction contrast tomography [32][33][34]. By contrast to FEM, the FFT method is limited to a periodic homogenisation scheme which makes it less general.

In the present work, we first investigate the application of the FFT method in the context of plasticity induced by diffusional transformation. Following previous investigation, an enhanced numerical scheme is employed incorporating the pre-hardening effect, which induces the back stress and thus the anisotropic effect on transformation plasticity. Then, use is made of FFT reference results on representative polycrystalline aggregates for a critical analysis of two existing analytical micromechanical models [15][17]. These analytical models are compared for varying material data (transformation expansion coefficient).

\section{Formulation of FFT-based numerical scheme}

The FFT numerical scheme proposed by Moulinec and Souquet [25] relies on the Green functions method to solve a periodic boundary value problem for heterogeneous media. For completeness, its formulation is first recalled. It offers a straightforward framework to consider stress-free strains related to the solid-state transformation process. Then, the constitutive local equations for crystalline plasticity and transformation kinetic are detailed.

2.1. Lippmann-Schwinger equation for periodic media 


\subsubsection{Formulation}

In the case of a periodic boundary problem, the local displacement can be divided into fluctuation and average terms such that:

$$
\boldsymbol{u}(\boldsymbol{x})=\boldsymbol{u}^{\prime}(\boldsymbol{x})+\bar{\varepsilon} \boldsymbol{x}
$$

where, $u^{\prime}(x)$ is a periodic displacement. The strain field reads:

$$
\boldsymbol{\varepsilon}(\boldsymbol{u}(\boldsymbol{x}))=\boldsymbol{\varepsilon}\left(\boldsymbol{u}^{\prime}(\boldsymbol{x})\right)+\bar{\varepsilon}
$$

with $\left\langle\boldsymbol{\varepsilon}\left(\boldsymbol{u}^{\prime}(\boldsymbol{x})\right)\right\rangle=0$.

For a heterogeneous elasto-plastic material, the rate-form of constitutive relation reads:

$$
\dot{\boldsymbol{\sigma}}(\boldsymbol{x})=\boldsymbol{C}(\boldsymbol{x}): \dot{\boldsymbol{\varepsilon}}^{e}(\boldsymbol{x})=\boldsymbol{C}(\boldsymbol{x}):\left(\dot{\boldsymbol{\varepsilon}}(\boldsymbol{x})-\dot{\boldsymbol{\varepsilon}}^{p}(\boldsymbol{x})-\dot{\boldsymbol{\varepsilon}}^{m}(\boldsymbol{x})\right)
$$

where $\boldsymbol{C}(x)$ is the elastic tensor at local position $x$ and $\boldsymbol{\varepsilon}^{e}, \boldsymbol{\varepsilon}, \boldsymbol{\varepsilon}^{p}$ and $\boldsymbol{\varepsilon}^{m}$ are elastic, total, plastic and transformation strain tensor respectively. Equivalently, the constitutive law can be rewitten:

$$
\dot{\sigma}(x)=\boldsymbol{C}(x): \dot{\varepsilon}(x)+\dot{\boldsymbol{\tau}}(x)
$$

By introducing homogeneous reference media with elasticity $\boldsymbol{C}^{0}$, equation (4) can be rewritten as:

$$
\begin{gathered}
\dot{\boldsymbol{\sigma}}(\boldsymbol{x})=\boldsymbol{C}^{0}: \dot{\boldsymbol{\varepsilon}}(\boldsymbol{x})+\left(\boldsymbol{C}(\boldsymbol{x})-\boldsymbol{C}^{0}\right): \dot{\boldsymbol{\varepsilon}}(\boldsymbol{x})+\boldsymbol{C}(\boldsymbol{x}):\left(\dot{\boldsymbol{\varepsilon}}^{p}(\boldsymbol{x})+\dot{\boldsymbol{\varepsilon}}^{m}(\boldsymbol{x})\right)=\boldsymbol{C}^{0}: \dot{\boldsymbol{\varepsilon}}(\boldsymbol{x})+\dot{\boldsymbol{\tau}}(\boldsymbol{x}) \\
\forall x \in V, \quad \operatorname{div} \dot{\boldsymbol{\sigma}}=0 \quad \forall x \in V, \quad \ddot{u} \#, \quad \dot{\boldsymbol{\sigma}} \cdot \boldsymbol{n}-\#
\end{gathered}
$$

where $\dot{\boldsymbol{\tau}}(\boldsymbol{x})$ is a polarisation tensor which comprises the contributions from the elastic heterogeneity, the plastic strain and transformation strains (eigenstrains). Solution of the problem reads:

$$
\dot{\boldsymbol{\varepsilon}}(\boldsymbol{x})=\dot{\bar{\varepsilon}}-\boldsymbol{\Gamma}^{\mathbf{0}} * \dot{\boldsymbol{\tau}}
$$

FFT-based iterative algorithms have been proposed to solve equation (6). In the following, use has been made of the fixed-point method initially proposed by [25].

Equations (5) in Fourier space are written as follows:

$$
\hat{\boldsymbol{\sigma}}(\xi)=\mathrm{i} \boldsymbol{C}^{0}:\left(\hat{\boldsymbol{u}}^{\prime}(\xi) \otimes \xi\right)+\hat{\boldsymbol{\tau}}(\xi), \quad \mathrm{i} \hat{\boldsymbol{\sigma}}(\xi) \cdot \xi=0
$$

where, $\xi$ is frequency, and the non-italic character i represents imaginary number. By eliminating $\hat{\boldsymbol{\sigma}}$ from equation (7), we obtain

$$
\hat{\dot{u}}^{\prime}(\xi)=\frac{\mathrm{i}}{2}\left(\boldsymbol{N}^{0} \otimes \xi+\xi \otimes \boldsymbol{N}^{0}\right) \hat{\boldsymbol{\tau}}(\xi)
$$

where,

$$
\begin{gathered}
\boldsymbol{N}^{0}(\xi)=\boldsymbol{K}^{0}(\xi)^{-1}, \boldsymbol{K}^{0}(\xi)=\boldsymbol{C}^{0}:(\xi \otimes \xi) \\
\hat{\boldsymbol{\varepsilon}}(\xi)=\frac{\mathrm{i}}{2}\left(\xi \otimes \hat{\boldsymbol{u}}^{\prime}(\xi)+\hat{\boldsymbol{u}}^{\prime}(\xi) \otimes \xi\right)=-\hat{\boldsymbol{\Gamma}}^{0}(\xi): \hat{\boldsymbol{\tau}}(\xi) \\
\hat{\boldsymbol{\Gamma}}^{0}(\xi)=\frac{1}{4}\left(N_{l i}^{0} \xi_{j} \xi_{k}+N_{k i}^{0} \xi_{j} \xi_{l}+N_{l j}^{0} \xi_{i} \xi_{k}+N_{k j}^{0} \xi_{i} \xi_{l}\right)
\end{gathered}
$$

$\hat{\boldsymbol{\Gamma}}^{0}$ is a periodic Green's operator. Inverse Fourier transformation of equation (10) gives the strain field within the heterogeneous medium.

The iterative algorithm to solve this problem is described below.

2.1.2. Iterative algorithm 
Initialisation: $\dot{\boldsymbol{\varepsilon}}^{0}=\dot{\boldsymbol{\varepsilon}}^{n}(\boldsymbol{x}), \forall x \in V$

Iteration: $(n+1): \dot{\boldsymbol{\varepsilon}}^{n}(\boldsymbol{x})$ and $\dot{\boldsymbol{\sigma}}^{n}(\boldsymbol{x})$ are known

(a) $\hat{\boldsymbol{\sigma}}^{n}=F F T\left(\dot{\boldsymbol{\sigma}}^{n}\right)$

(b) Check convergence

$e^{n}=\sqrt{\left\langle\left\|\operatorname{div}\left(\dot{\boldsymbol{\sigma}}^{n}\right)\right\|^{2}\right\rangle}=\sqrt{\left\langle\left\|\xi \cdot \hat{\boldsymbol{\sigma}}^{n}(\xi)\right\|^{2}\right\rangle}<\epsilon \quad$ if no macroscopic stress is imposed

$e^{n}=\frac{\sqrt{\left.\left\|\operatorname{div}\left(\dot{\boldsymbol{\sigma}}^{n}\right)\right\|^{2}\right\rangle}}{\left\|\left\langle\dot{\boldsymbol{\sigma}}^{n}\right\rangle\right\|}=\frac{\sqrt{\left.\|\| \xi \hat{\boldsymbol{\sigma}}^{n}(\xi) \|^{2}\right\rangle}}{\left\|\hat{\boldsymbol{\sigma}}^{n}(\mathbf{0})\right\|}<\epsilon \quad$ otherwise

(c) $\hat{\dot{\boldsymbol{\varepsilon}}}^{n+1}(\xi)=\hat{\dot{\boldsymbol{\varepsilon}}}^{n}(\xi)-\hat{\boldsymbol{\Gamma}}^{0}(\xi): \hat{\boldsymbol{\sigma}}^{n}(\xi) \quad \forall \xi \neq 0 \quad$ and $\quad \hat{\dot{\boldsymbol{\varepsilon}}}^{n+1}(0)=\dot{\bar{\varepsilon}}$

(d) $\dot{\boldsymbol{\varepsilon}}^{n+1}=F F T^{-1}\left(\hat{\dot{\varepsilon}}^{n+1}\right)$

(e) $\dot{\boldsymbol{\sigma}}^{n+1}(\boldsymbol{x})=g\left(\dot{\boldsymbol{\varepsilon}}^{n+1}(\boldsymbol{x})\right) \quad \forall x \in V$

where, $g$ is a constitutive equation which relates strain and stress (equation (3), in the present case).

In the case where the macroscopic stress is imposed, the procedure (c) is modified as:

(c') $\hat{\dot{\boldsymbol{\varepsilon}}}^{n+1}(\xi)=\hat{\dot{\boldsymbol{\varepsilon}}}^{n}(\xi)-\hat{\boldsymbol{\Gamma}}^{0}(\xi): \hat{\boldsymbol{\sigma}}^{n}(\xi) \quad \forall \xi \neq 0 \quad$ and $\hat{\dot{\boldsymbol{\varepsilon}}}^{n+1}(0)=\left\langle\boldsymbol{\varepsilon}^{n}\right\rangle+\boldsymbol{C}^{\mathbf{0}-1}:\left(\overline{\boldsymbol{\sigma}}^{n}-\left\langle\boldsymbol{\sigma}^{n-1}\right\rangle\right)$.

\subsection{Constitutive behaviour, microstructure and phase transformation}

\subsubsection{Constitutive equations by crystal plasticity}

Plastic strain is the result of dislocation glide. The slip occurs along certain directions on certain planes. Such combinations of directions and planes are called slip systems. Let $\dot{\gamma}^{\alpha}$ be the slip rate on the $\alpha$ slip system. The plastic strain rate is the determined by the sum of slip rates on all slip systems:

$$
\dot{\boldsymbol{\varepsilon}}^{p}=\sum_{\alpha} \boldsymbol{p}^{\alpha} \dot{\gamma}^{\alpha}
$$

where $\boldsymbol{p}^{\alpha}$ is the Schmid tensor of $\alpha$ slip system.

$$
\boldsymbol{p}^{\alpha}=\frac{1}{2}\left(\boldsymbol{s}^{\alpha} \otimes \boldsymbol{m}^{\alpha}+\boldsymbol{m}^{\alpha} \otimes \boldsymbol{s}^{\alpha}\right)
$$

$\boldsymbol{s}^{\alpha}$ and $\boldsymbol{m}^{\alpha}$ are respectively the slip direction and slip plane normal of the $\alpha$ slip system. According to the Schmid law, the slip system becomes active when the resolved shear stress $\tau^{\alpha}$ becomes equal to the Critical Resolved Shear Stress (CRSS) $g^{\alpha}$ :

$$
\left|\tau^{\alpha}\right|=\left|\boldsymbol{p}^{\alpha}: \boldsymbol{\sigma}\right|=g^{\alpha}
$$

During elasto-plastic deformation, the equation (14) is satisfied. In other words, stress increases according to the hardening of each slip system.

For such a rate-independent problem, Hutchinson [35] proposed the following solution for determining the slip rate of each slip system by using the consistence condition:

$$
\dot{\tau}^{\alpha}=\operatorname{sgn}\left(\tau^{\alpha}\right) \dot{g}^{\alpha}
$$

For the small deformation problems, equation (15) can be rewritten as: 


$$
\dot{\boldsymbol{\sigma}}: \boldsymbol{p}^{\alpha}=\sum_{\beta} h^{\alpha \beta} \dot{\gamma}^{\beta}
$$

and the general form of the hardening law is:

$$
\dot{g}^{\alpha}=\sum_{\beta} h^{\alpha \beta}\left|\dot{\gamma}^{\beta}\right|
$$

with $h^{\alpha \beta}$ the hardening coefficients. They can be expressed phenomenologically with an additional evolution law. We adopt the following form [36]:

$$
\begin{gathered}
h^{\alpha \alpha}=h=H_{0} \operatorname{sech}^{2}\left[\frac{H_{\beta} \sum_{\beta} \gamma^{\beta}}{\tau_{s}-\tau_{0}}\right] \\
h^{\alpha \beta}=q h+(1-q) h \delta_{\alpha \beta}
\end{gathered}
$$

where $H_{0}$ is a coefficient parameter, $\tau_{0}$ is the initial yield stress value and $\tau_{s}$ is the saturated stress value. The parameter $q$, which describes latent hardening, takes values from 1.0 to 1.4 depending on the material.

By using the constitutive laws, one obtains.

$$
\dot{\boldsymbol{\sigma}}: \boldsymbol{p}^{\alpha}=\boldsymbol{C}:\left(\dot{\boldsymbol{\varepsilon}}-\dot{\boldsymbol{\varepsilon}}^{p}-\dot{\boldsymbol{\varepsilon}}^{t h}-\dot{\boldsymbol{\varepsilon}}^{m}\right): \boldsymbol{p}^{\alpha}=\boldsymbol{C}:\left(\dot{\boldsymbol{\varepsilon}}-\dot{\boldsymbol{\varepsilon}}^{t h}-\dot{\boldsymbol{\varepsilon}}^{m}\right): \boldsymbol{p}^{\alpha}-\sum_{\beta} \boldsymbol{p}^{\alpha}: \boldsymbol{C}: \boldsymbol{p}^{\beta} \dot{\gamma}^{\beta}=\sum_{\beta} h^{\alpha \beta} \dot{\gamma}^{\beta}
$$

and the slip rates are expressible as:

$$
\dot{\gamma}^{\alpha}=\boldsymbol{f}^{\alpha}:\left(\dot{\boldsymbol{\varepsilon}}-\dot{\boldsymbol{\varepsilon}}^{t h}-\dot{\boldsymbol{\varepsilon}}^{m}\right) \quad \text { with } \quad \boldsymbol{f}^{\alpha}=\sum_{\beta} Y^{\alpha \beta} \boldsymbol{C}: \boldsymbol{p}^{\beta}
$$

with

$$
Y^{\alpha \beta}=\left(X^{\alpha \beta}\right)^{-1} \text { and } X^{\alpha \beta}=h^{\alpha \beta}+\boldsymbol{p}^{\alpha}: \boldsymbol{C}: \boldsymbol{p}^{\beta}
$$

The matrix $X^{\alpha \beta}$ has to be non-singular. For that reason, the maximum number of active slip systems, i.e. rank of the matrix $X^{\alpha \beta}$, is limited to 5. From equation (21), we can find the value of the shear strain rates, which in turn depends on the prescribed strain rate or stress rate.

For the elasto-plastic transition problem, initial values of $\tau^{\alpha}$ and $g^{\alpha}$ are generally different. The incremental form of equation (15) during a finite time increment can be written as follows:

$$
\tau^{\alpha}+\Delta \tau^{\alpha}=\operatorname{sgn}\left(\tau^{\alpha}\right)\left(g^{\alpha}+\Delta g^{\alpha}\right)
$$

Note that $\tau^{\alpha}$ and $g^{\alpha}$ are the values evaluated at the previous step. $\Delta$ represents one increment during one time step.

Consequently, equation (16) takes the form:

$$
(\boldsymbol{\sigma}+\Delta \boldsymbol{\sigma}): \boldsymbol{p}^{\alpha}=\operatorname{sgn}\left(\tau^{\alpha}\right) g^{\alpha}+\sum_{\beta} h^{\alpha \beta} \Delta \gamma^{\beta}
$$

Following the previous discussion, the plastic slip increments can be expressed as (see Appendix A):

$$
\Delta \gamma^{\alpha}=\sum_{\beta} Y^{\alpha \beta}\left(\boldsymbol{\sigma}: \boldsymbol{p}^{\beta}+\boldsymbol{p}^{\beta}: \boldsymbol{C}:\left(\Delta \boldsymbol{\varepsilon}-\Delta \boldsymbol{\varepsilon}^{t h}-\Delta \boldsymbol{\varepsilon}^{m}\right)-\operatorname{sgn}\left(\tau^{\beta}\right) g^{\beta}\right)
$$


If the resolved stress equates CRSS at the previous step, then the equation (25) becomes exactly equivalent to as equation (21).

\subsubsection{Microstructures}

A cubic area volume with regularly allocated calculation points (voxels) is divided into cells (grains). This procedure is realised by using the Voronoi tessellation method. First, grain seeds are spread inside the cube. The number of the grains is thus fixed at the beginning of the calculation. Then, the surfaces which have same distances from two neighbouring grain seeds are considered to be flat grain boundaries between these two grains.

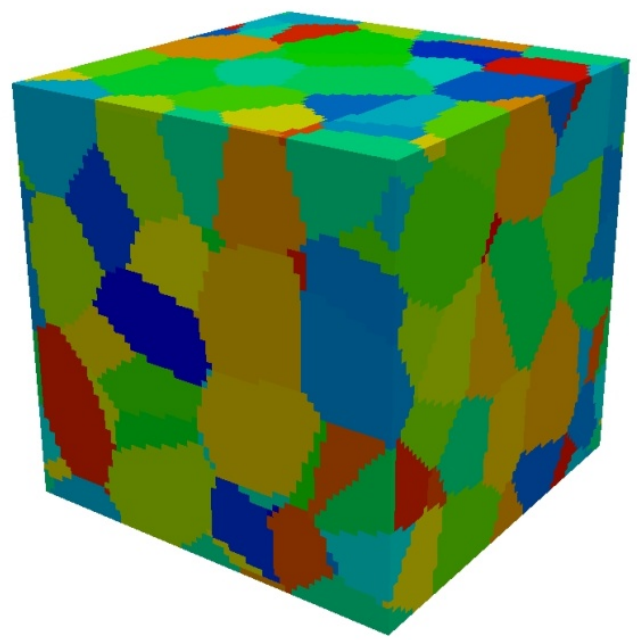

(a) Initial austenite phase

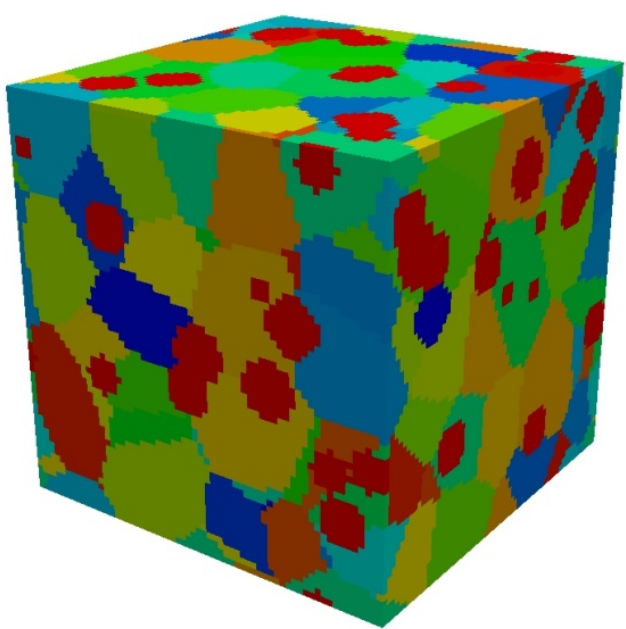

(b) During phase transformation

Fig. 1 Sample Voronoi tessellation and phase transformation; 100 grains in $64^{3}$ cubic space.

All voxels which are included into one grain have the same crystallographic orientation. Hereafter, initial grain seeds and initial crystallographic orientations of each grain are randomly distributed. The heterogeneity of the polycrystal then derives solely from the crystalline orientation distributions together with the local anisotropy of the single crystal behaviour.

\subsubsection{Phase transformation model}

Phase transformation is considered here to be diffusive, isotropic and isothermal. As a consequence, there are no thermal strains within the polycrystal. This simplifies the problem and provides the basic results of transformation plasticity. In accordance with the numerical homogenisation scheme with periodic displacement boundaries, the periodicity of the phase transformation has to be imposed. One example is shown in Fig. 1 (b); the 100 initial grains and 100 growing new grains (red coloured circles).

A finite intermediate zone between parent and daughter phases, where the volume fraction of new phase takes value of $0 \leq \xi \leq 1$, is defined at the circumference of the daughter phase particles. 
Within this intermediate zone, the volume fraction of the daughter phase obeys KJMA (Kolmogolov-Johnson-Mehl-Avrami) type equation [37]:

$$
\xi=1-\exp \left(-b t^{n}\right)
$$

where $b$ and $n$ are temperature dependent material parameters defining the intermediate zone thickness. When the frontier of a new grain reaches a calculation point, the value of time $t$ in equation (26) is set to be zero and the transformation begins according to the equation.

When the volume fraction exceeds $50 \%$, the constitutive behaviour of the daughter phase is considered to be transformed and being a new phase. At the same time, the crystallographic orientation is changed according to Kurdjumow-Sachs (K-S) relation [38]. There are 24 possible variants for the K-S relation; in the present calculation, one of these 24 variants is randomly selected for the new grain.

The calculations start with $100 \%$ parent grains which are subject to a constant uniaxial stress loading along the z-axis. The constant applied stress value takes values between $-90 \mathrm{MPa}$ and 90 MPa is held until phase transformation is complete, i.e. the calculation reaches $100 \%$ of daughter phase throughout the cubic region.

\subsection{Applications}

\subsubsection{Validation of the numerical model}

In order to validate the FFT-based model, the numerical results have been assessed by comparison with the exact theoretical result of Leblond [14] for an isotropic polycrystalline aggregate undergoing phase transformation without macroscopic applied stress.

In this particular case, it has been shown that the overall plastic strain is necessarily null. As a consequence, the exact macroscopic strain of the polycrystal reads

$$
\bar{\varepsilon}=\bar{\varepsilon}^{m}=\beta \xi
$$

with $\xi$, the macroscopic volume fraction of daughter phase. The results obtained for an austenitic polycrystal composed of 100 grains with random orientations are shown in Fig. 2. Note that this case uses the same elastic constants for each phase. The numerical response agrees with the exact result. The chosen numerical microstructure can thus be considered isotropic. It is noted that there is no macroscopic plastic strain despite the plastic deformation occurs at the crystal (microscopic) level. 


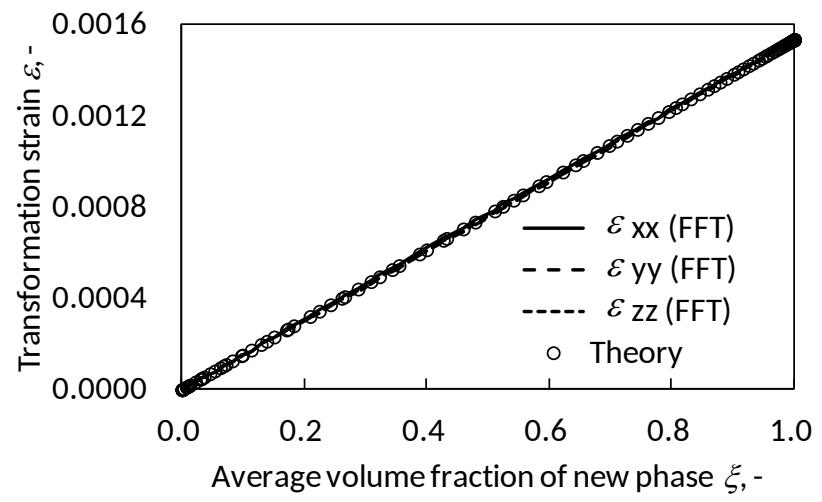

Fig. 2

Evolution of the macroscopic strain with the volume fraction of transformed phase (no applied stress, isotropic polycrystal).

\subsubsection{Calculation of transformation plasticity}

In the present study, the mechanical behaviour of carbon steel undergoing austenite-pearlite phase transformation will be investigated. The set of plasticity parameters for this steel grade is shown in Table 1. The macroscopic stress/strain relation of austenite phase and pearlite phase are calculated by using FFT model and are depicted in Fig. 3. The selected material parameters have been adjusted in order to get a good fit between FFT calculations and experimental data (the experimental data corresponding to the austenite phase are also shown in Fig. 3)

Table 1 Parameters for crystal plasticity calculation.

\begin{tabular}{|c|c|c|}
\hline & Austenite & Pearlite \\
\hline Bulk modulus $(\mathrm{MPa})$ & 135833 & 150000 \\
\hline Shear modulus $(\mathrm{MPa})$ & 62692 & 69231 \\
\hline$H_{0}(\mathrm{MPa})$ & 10 & 550 \\
\hline$\tau_{0}(\mathrm{MPa})$ & 30 & 100 \\
\hline$\tau_{s}(\mathrm{MPa})$ & 40 & 130 \\
\hline$q$ & 1.0 & 1.0 \\
\hline Number of grains & 100 & 100 \\
\hline
\end{tabular}




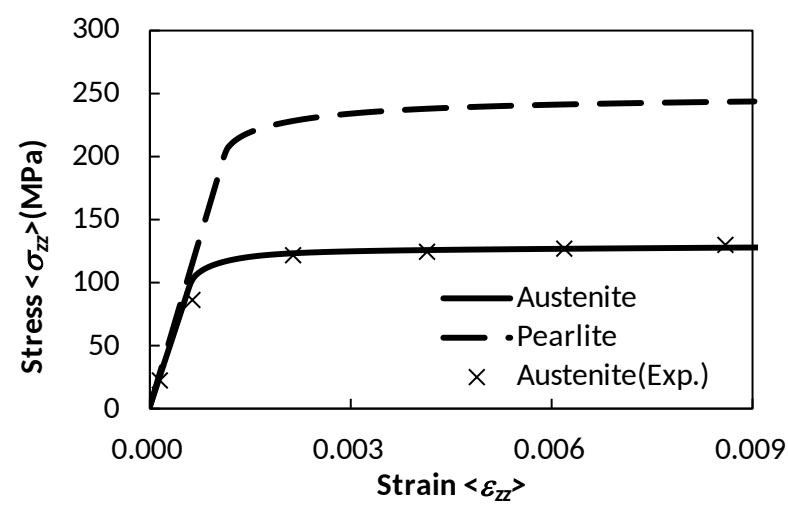

Fig. 3

Macroscopic stress/strain curves of Austenite and Pearlite phase (calculations and experiment at $670^{\circ} \mathrm{C}$ ).

In order to analyse thoroughly each model, two cases of transformation expansion coefficients will be considered $\left(\beta=1.5 \times 10^{-3}\right.$ and $\left.\beta=4.4 \times 10^{-3}\right)$. The new phase grain seeds are spread randomly and consequently, subsequent isotropic grain growth starts.

Case of small transformation expansion $\left(\beta=1.5 \times 10^{-3}\right)$

The stress-strain calculation during phase transformation is performed in a reference volume element with periodic boundaries. In this procedure, the equivalent plastic strain accumulation is pronounced at the vicinity of the daughter phase as demonstrated in Fig. 4.

The evolution of the average volume fraction of the new phase under stress free condition is shown in Fig. 5 (a). Within the period of phase transformation, the strain values change according to the magnitude of applied stress (transformation plastic strain). This additional strain effect (transformation plasticity) is depicted in Fig. 5 (b). As the applied stress value increases, the resulting total strain value also increases. As seen below, transformation plasticity is naturally obtained at the macroscopic scale during phase transformation as a result of the scale-transition numerical approach. 


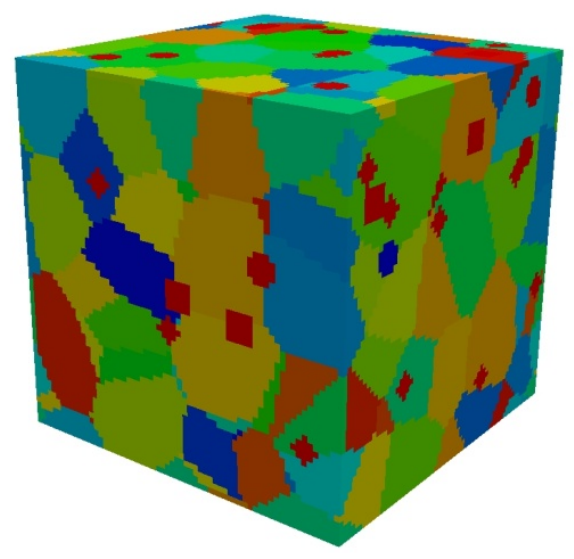

(a) phase distribution (3\%)

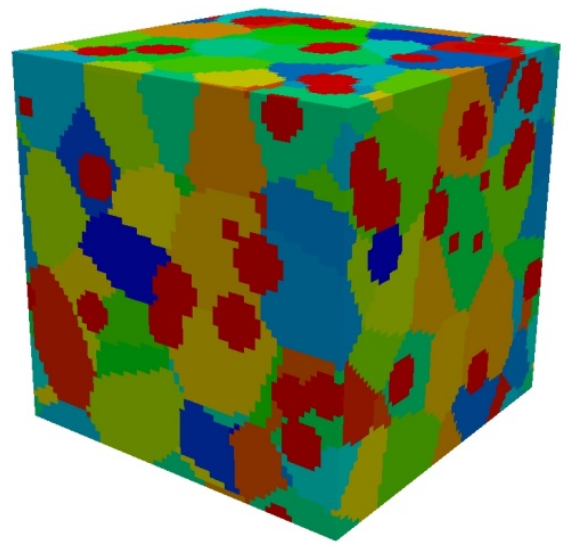

(c) phase distribution $(10 \%)$

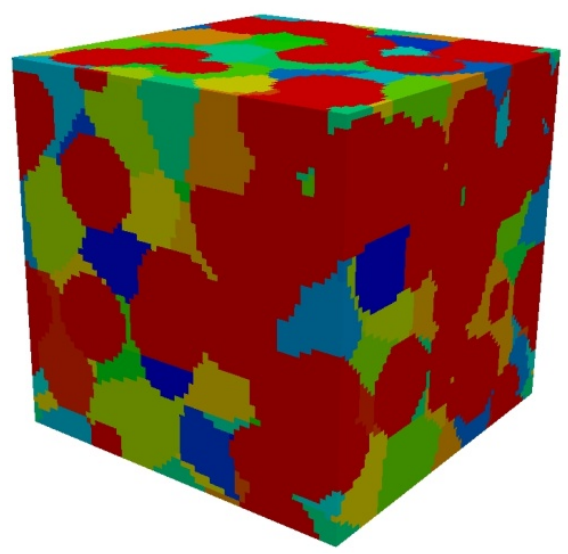

(e) phase distribution (50\%)

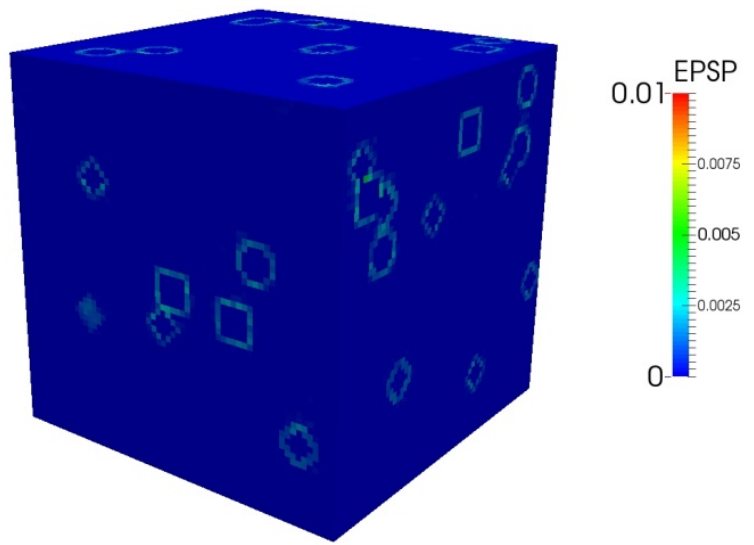

(b) equivalent plastic strain in mother phase (3\%)

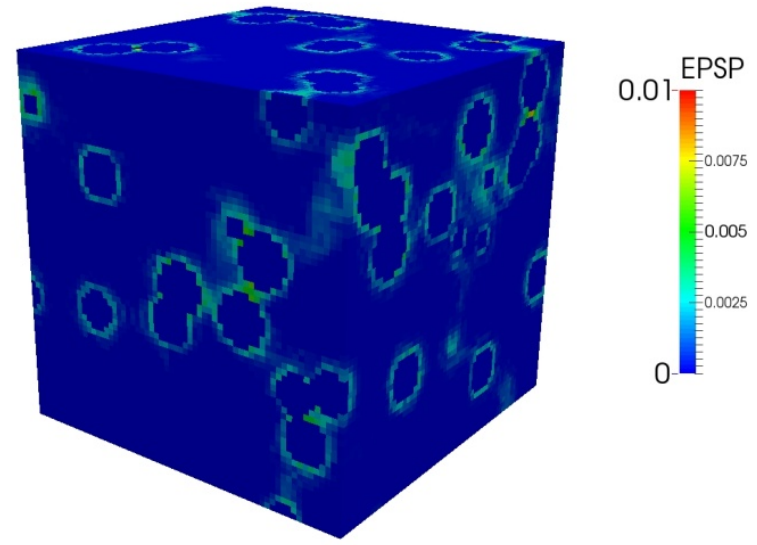

(d) equivalent plastic strain in mother phase (10\%)
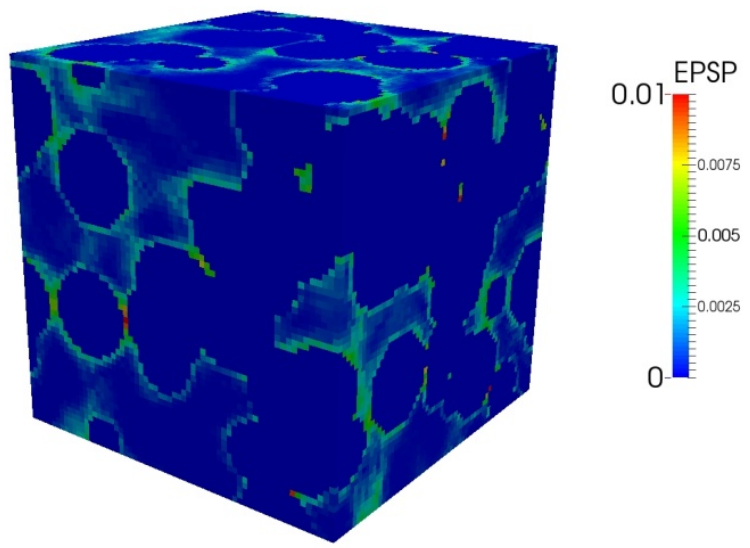

(f) equivalent plastic strain in mother phase (50\%)

Fig. 4 Evolution of volume fraction of new phase and macroscopic total strain. 


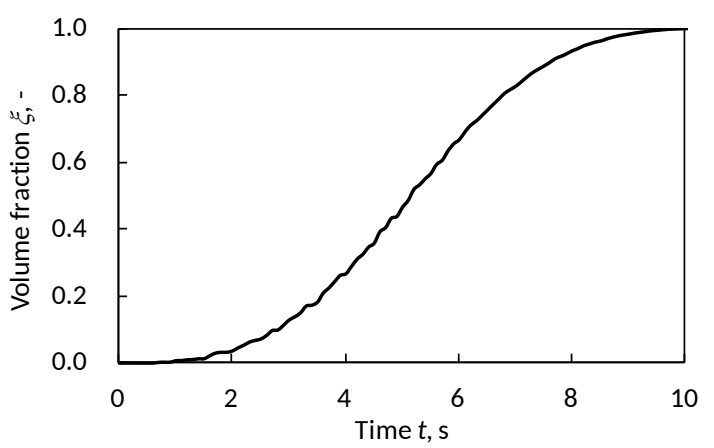

(a) Evolution of volume fraction

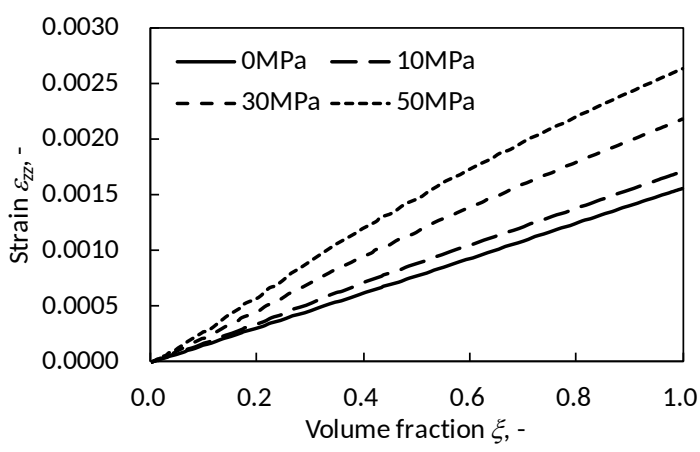

(b) Evolution of total strain

Fig. 5 Evolution of volume fraction of new phase and macroscopic total strain.

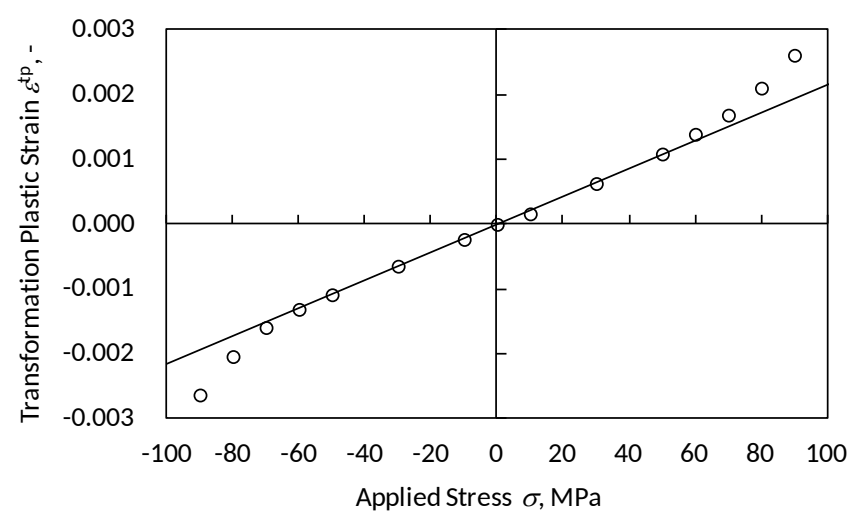

Fig. 6 Relation between applied stress and transformation plastic strain.

The transformation plastic strain after phase transformation is plotted in Fig. 6 as a function of the applied stress. One can observe in the Fig. 6 that there is indeed a linear relation between applied stress and transformation plastic strain when applied stress is less than $60 \mathrm{MPa}$. This relation agrees with the predictions of the models by Greenwood-Johnson [2], Leblond [14] or Inoue [11]. The factors of proportionality (transformation plasticity coefficient) are equal to $K_{P}^{+}=2.20 \times 10^{-5}$ $\left(\mathrm{MPa}^{-1}\right)$ and $K_{P}^{-}=2.15 \times 10^{-5}\left(\mathrm{MPa}^{-1}\right)$, where the superscripts "+" and "-" denote transformation plasticity under tensile applied stress and compressive applied stress respectively. Thus, there is no significant difference between tensile and compressive applied stresses in the present case. To make a quantitative comparison with Leblond's model, the transformation plasticity coefficient by Leblond's model is $K_{P}=2.17 \times 10^{-5}\left(\mathrm{MPa}^{-1}\right)$; a good agreement between the two approaches. We can then conclude that the FFT model is able to predict correctly the transformation plasticity due to a diffusive Greenwood-Johnson process.

In addition to the proportional relationship between applied stress and transformation plastic strain, the results by FFT predict satisfactorily the experimental evidence of non linearity between applied 
stress and transformation plastic strain when applied stress is relatively large.

Case of large transformation expansion $\left(\beta=4.4 \times 10^{-3}\right)$

The large transformation strain induced plasticity is analysed. All the conditions except the transformation strain are the same as those used for the small transformation expansion case. Fig. 7 is the transformation plastic strain calculated by FFT numerical model depending on the applied constant stresses.

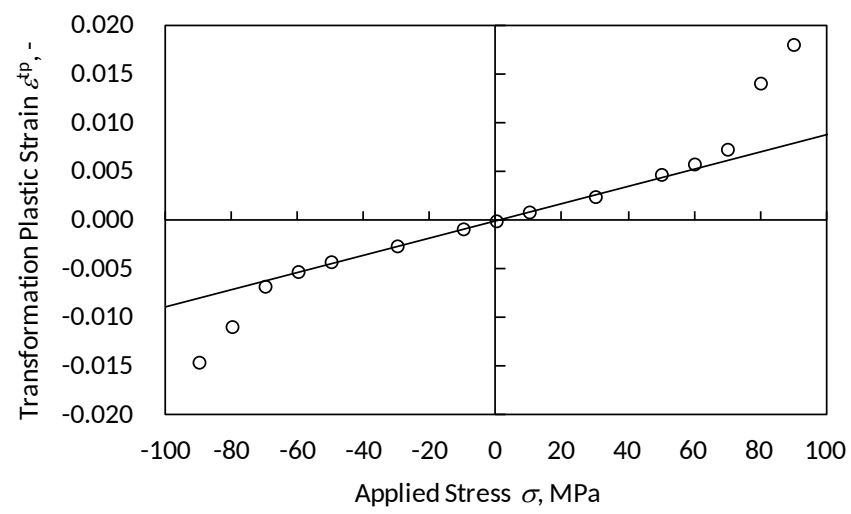

Fig. 7 Relation between applied stress and transformation plastic strain.

From the obtained data, the transformation plastic coefficient is $K_{P}^{+}=8.44 \times 10^{-5}\left(\mathrm{MPa}^{-1}\right)$ for tensile and $K_{P}^{-}=9.38 \times 10^{-5}\left(\mathrm{MPa}^{-1}\right)$ for compressive applied stress. There is a slight discrepancy between transformation plastic coefficients under tensile and compressive stresses but the difference is below $10 \%$ even in the large transformation expansion case. The nonlinearity behaviour for large applied stress is found to be more pronounced than in the small transformation expansion case.

\subsubsection{Pre-deformation effect on transformation strains}

Heat treatment process in steels is often accompanied with pre-deformation, such as hot rolling and hot forging. Hence, the effect of pre-deformation is inherently linked to the precise shape and residual stress analyses. As a basic analysis, let us conduct a uniaxial tension simulation followed by phase transformation introducing back stress effect on each slip system for the following simulations, such that

$$
\tau^{*(\alpha)}=\tau^{(\alpha)}-a^{(\alpha)}
$$

where $\tau^{*(\alpha)}$ is effective resolved shear stress and $a^{(\alpha)}$ is back stress value on the $\alpha$ slip system. Now let $a^{(\alpha)}$ be Armstrong-Frederick type back stress model [39], such that:

$$
\dot{a}^{(\alpha)}=C_{1}\left(C_{2} \dot{\gamma}^{(\alpha)}-a^{(\alpha)}\left|\dot{\gamma}^{(\alpha)}\right|\right)
$$

where $C_{1}$ and $C_{2}$ are material constants. 
A uniaxial tensile calculation up to $10 \%$ strain of polycrystalline aggregates is conducted followed by phase transformation calculation under applied constant stress. This sequence is depicted in Fig. 8. At 1000 step (10\% strain), the unloading process to a prescribed stress is calculated and then the phase transformation calculation is initiated.

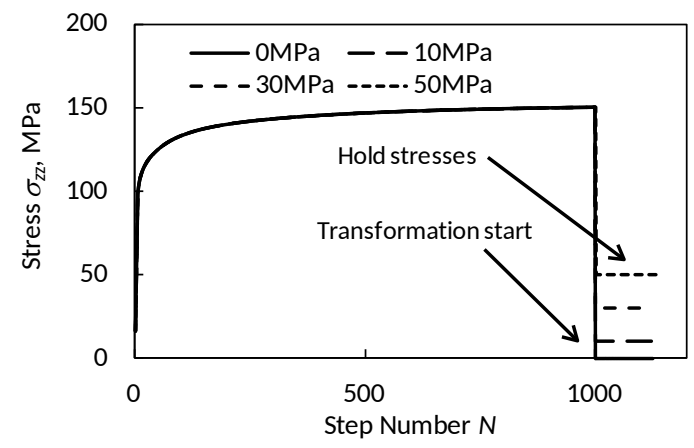

Fig. 8 Calculation sequence for pre-tension transformation plasticity.

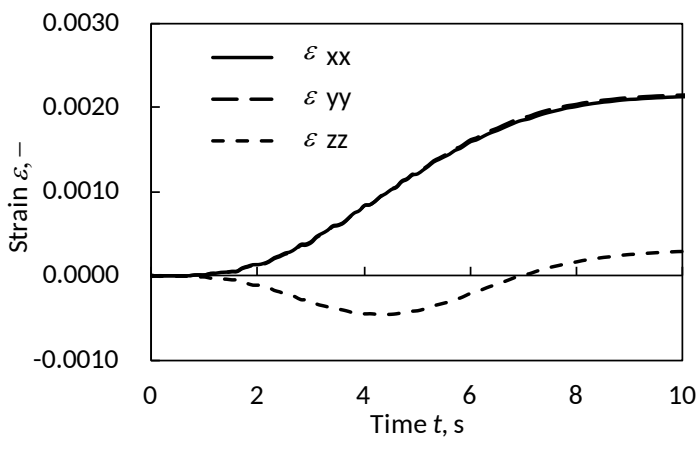

Fig. 9 Transformation strain with pre-tension.

The obtained transformation strains under free applied stress are demonstrated in Fig. 9. A remarkable diverge of $\varepsilon_{z z}$ (pre-tensile direction) from the other strain components is observed. The strain $\varepsilon_{z z}$ takes minus value at the beginning of transformation even though the transformation is an expanding process. The other two components take the same larger value. Obviously, this diverge is controlled by the classical plastic strain and thus the total transformation strain (volume change) keeps constant from the input value. In fact, $\varepsilon_{i i}^{m}=\varepsilon_{11}^{m}(=0.002146)+\varepsilon_{22}^{m}(=0.002153)+\varepsilon_{33}^{m}$ $(=0.000296)=0.004595 \cong 3 \times \beta(=0.001536)$. The mechanism of this remarkable phenomenon can be given as follows.

During pre-tension, the yield surface moves, and thus material gets hardened for the loading direction. By contrast, the material is softer after deformation for the opposite direction exhibiting anisotropy. To accommodate the volume difference between parent and daughter phases, as we have 
seen in the previous section, plastic strain occurs at the vicinity of the daughter phase. If the material is isotropic, this plastic strain gives no apparent macroscopic strain. However, it is not applicable for the anisotropic materials. The present case, the anisotropy in the RVE is induced by the pre-tensile calculation. Therefore, it is hard to deform in the tensile direction resulting in the small (even negative because of the deformation in the other directions) plastic strain yielding large plastic strain in the other directions (because the incompressive plastic deformation). This phenomenon can also be found in the experimental works by Taleb et al. [10] in bainitic phase transformation in 16MND5 steel. The experimental results appear to be very similar to the calculations. Above explanation is also valid for the pre-compressed case. To confirm this, $-10 \%$ pre-compressed followed by unloading and phase transformation calculations are carried out. The calculated results of free dilatation are shown in Fig. 10. It is observed that the total strain in $z$ direction is larger than those of the other directions even though the local transformation strain is always isotropic. Again, the total volume change remains the same value with that of non-hardening condition.

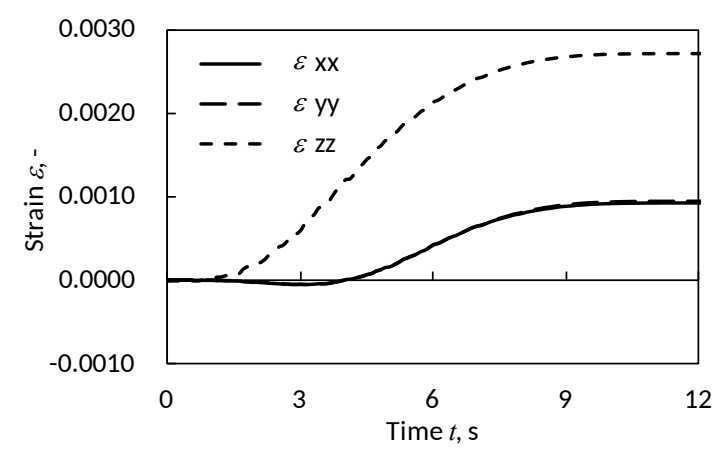

Fig. 10 Transformation strain with pre-compression.

In analogy with the previous calculations, following pre-hardening, strains under several applied stresses during phase transformation are calculated. In this case, the transformation plastic strain depending on the applied stress is plotted in Fig. 11.

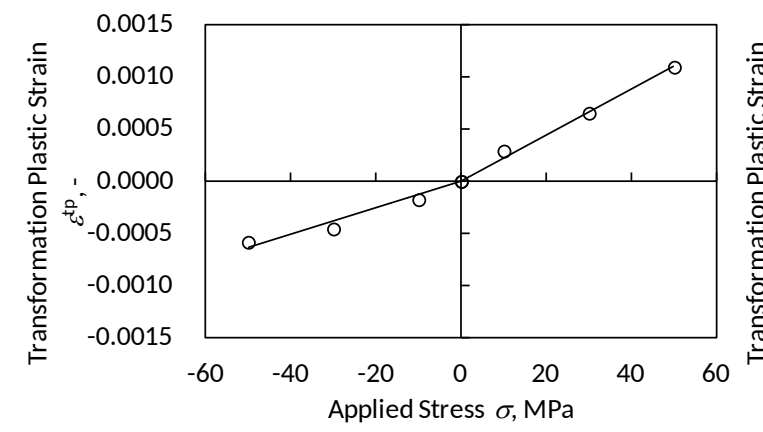

(a) pre-tension

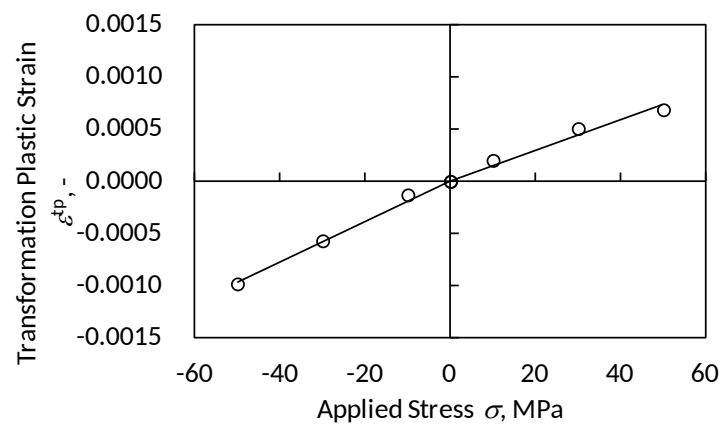

(b) pre-compression 
Fig. 11 Relation between transformation plastic strain and applied stress.

For small applied stresses (smaller than $60 \mathrm{MPa}$ ), the proportional factors (transformation plastic coefficients) of each case are given by follows.

Pre-tension :

$$
\begin{aligned}
& K_{P}^{+}=2.12 \times 10^{-5} \mathrm{MPa}^{-1}, K_{P}^{-}=1.17 \times 10^{-5} \mathrm{MPa}^{-1} \\
& K_{P}^{+}=1.36 \times 10^{-5} \mathrm{MPa}^{-1}, K_{P}^{-}=2.01 \times 10^{-5} \mathrm{MPa}^{-1}
\end{aligned}
$$

Pre-compression:

The superscript of "+" represents positive applied constant applied stress during phase transformation, and “-” represents negative applied stress. It is found that the linear relation between applied stress and transformation plastic strain is kinked because of the pre-hardening effect.

\section{Assessment of existing analytical models}

\subsection{Descriptions of selected existing analytical models}

\subsubsection{Leblond's model}

Leblond has developed an analytical model which allows to calculate the plastic strain state of the parent phase which surrounds a spherical inclusion of the daughter phase [15]. According to the Greenwood-Johnson mechanism, parent phase crust expansion by phase transformation induces some plastic strain in the surrounding parent phase. Leblond expressed the transformation plastic strain rate as a function of the volume fraction change, neglecting plastic strain in the daughter phase, such that,

$$
\dot{\varepsilon}^{t p}=(1-\xi)\left|\frac{\delta \varepsilon_{1}^{p}}{\delta \xi}\right|_{V_{1}} \dot{\xi}
$$

where $\dot{\varepsilon}^{t p}$ is equivalent plastic strain rate caused by transformation plasticity and $\left\langle\delta \varepsilon_{1}^{p} / \delta \xi\right\rangle_{V_{1}}$ represents the average value within the parent phase of the ratio of plastic strain increment due to volume fraction change. Starting with the equation (30), it is assumed that for small or moderately high applied stresses, the parent phase is entirely elastoplastic whereas the daughter phase remains elastic (or its plastic strain rate remains always much smaller than that of the parent phase).

Using Prandtl-Reuss relation, the equation (30) can be rewritten as:

$$
\dot{\varepsilon}^{t p}=\frac{3(1-\xi)}{2 \sigma_{1}^{y}}\left(\frac{\delta \varepsilon_{1}^{e q}}{\delta \xi}\right)_{V_{1}} S \dot{\xi}
$$

where $S$ is the applied macroscopic deviatoric stress value. The total equivalent strain increment due to volume change can be obtained by neglecting external stress effects 


$$
\left(\frac{\delta \varepsilon_{1}^{e q}}{\delta \xi}\right)_{V_{1}}=-\frac{2 \beta}{1-\xi} \ln (\xi)
$$

Finally we obtain

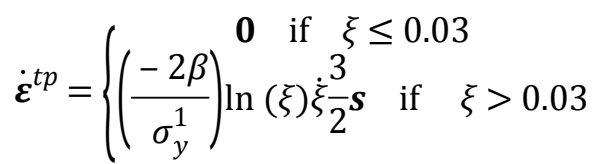

The total value of transformation plastic strain under constant applied stress is

$$
\boldsymbol{\varepsilon}^{t p}=\int_{0.03}^{1}\left(\frac{-2 \beta}{\sigma_{y}^{1}}\right) \ln (\xi) \dot{\xi} \frac{3}{2} \boldsymbol{s} d \xi \cong 0.8648\left(\frac{-2 \beta}{\sigma_{y}^{1}}\right)_{\frac{3}{2}}^{3} \boldsymbol{s}
$$

This model has been shown by Leblond to estimate correctly the transformation plastic strain in comparison with the experimental results. An extended model to strain hardening materials is suggested [16], namely,

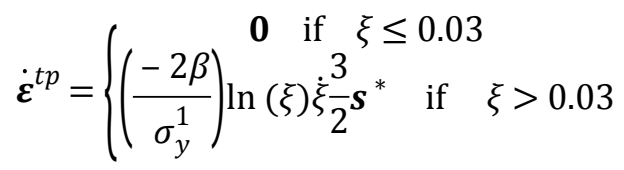

where $\boldsymbol{s}^{*}$ is a deviatoric effective stress; the deviatoric back stress is extracted from the deviatoric stress.

As we have seen above, transformation plasticity models are often expressed as a linear relationship between applied stress and transformation plastic strain. This is confirmed by many experimental works [11][40][41]. However, when the applied stress exceeds a certain threshold value, the linear relation does not hold anymore. The threshold value is often set equal to be a half of the value of yield stress value of weaker phase [2][42]. To the authors' knowledge, no theoretical or numerical explanation of this threshold value has been provided yet. However, a phenomenological modelling of the transformation plasticity including non-linear regime has been given by Leblond [16]:

$$
\dot{\boldsymbol{\varepsilon}}^{t p}=\left\{\left(\frac{-3 \beta}{\sigma_{y}^{1}}\right) h\left(\frac{\sigma^{e q}}{\sigma^{u}}\right) \cdot \ln (\xi) \dot{\xi} \boldsymbol{s} \text { if } \quad \xi>0.03\right.
$$

with

$$
h\left(\frac{\sigma^{e q}}{\sigma^{u}}\right)=\left\{\begin{array}{c}
1 \text { if } \frac{\sigma^{e q}}{\sigma^{u}} \leq \frac{1}{2} \\
1+3.5\left(\frac{\sigma^{e q}}{\sigma^{u}}-\frac{1}{2}\right) \text { if } \frac{\sigma^{e q}}{\sigma^{u}}>\frac{1}{2}
\end{array}\right.
$$

where the function $h\left(\sigma^{e q} / \sigma^{u}\right)$ expresses the nonlinearity, $\sigma^{e q}$ is the applied global equivalent stress 
and $\sigma^{u}$ is the global yield stress of the parent phase. The function $h$ is fitted with the results by FEM calculations.

\subsubsection{Taleb's model}

Taleb et al. (Taleb and Sidoroff, 2003) modified the Leblond's model by removing the hypothesis of entirely plastic parent phase, such that:

$$
\dot{\boldsymbol{\varepsilon}}^{t p}=\left\{\begin{array}{lll}
\left(\frac{-2 \beta}{\sigma_{y}^{1}}\right) \ln \left(\xi_{l}\right) \dot{\xi} \frac{3}{2} s & \text { if } & \xi \leq \xi_{l} \\
\left(\frac{-2 \beta}{\sigma_{y}^{1}}\right) \ln (\xi) \dot{\xi} \frac{3}{2} \boldsymbol{s} & \text { if } & \xi>\xi_{l}
\end{array}\right.
$$

with

$$
\xi_{l}=\frac{\sigma_{1}^{y} 4 \mu+3 K}{2 \beta 9 K \mu}
$$

where $K$ and $\mu$ are bulk and shear elastic moduli respectively. Thus, Taleb's model does not restrict the parent phase to be entirely elastoplastic until the volume fraction of daughter phase reaches the value indicated by equation (39). Consequently, Taleb's model is expected to be more accurate during the beginning of phase transformation. After the volume fraction of daughter phase becomes $\xi_{l}$, this model follows Leblond's model.

\subsection{Comparison of the analytical models with FFT numerical results}

First, transformation plastic strain for the small transformation expansion case $\left(\beta=1.5 \times 10^{-3}\right)$ by analytical solutions under several applied stresses is plotted and confronted with FFT numerical results in Fig. 12. Note that the line "Leblond" signifies Leblond's linear equation (33) and "Leblond-Mod." is non-linear equation (35). "Leblond-Mod." is an enhanced "Leblond" model comprises the nonlinearity under high external stress. All these models predict approximately the same transformation plastic strain values when applied stress is relatively small, whilst Leblond's modified model overestimates transformation plastic strain at large applied stresses. The mechanism of this nonlinearity will be discussed in the later section.

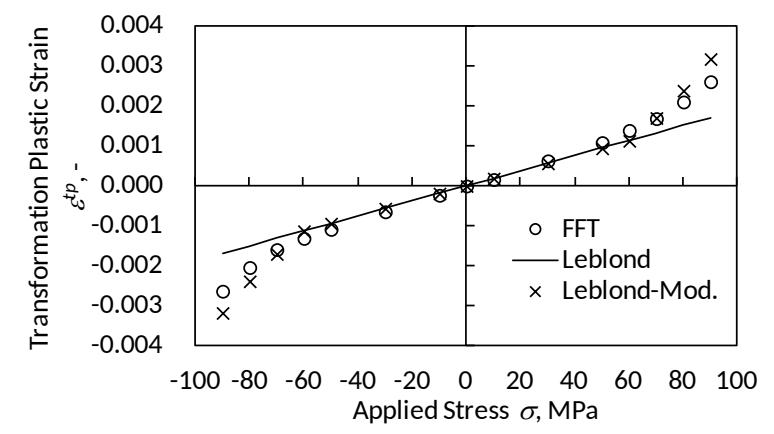

Fig. 12 Relation between applied stress and transformation plastic strain. 


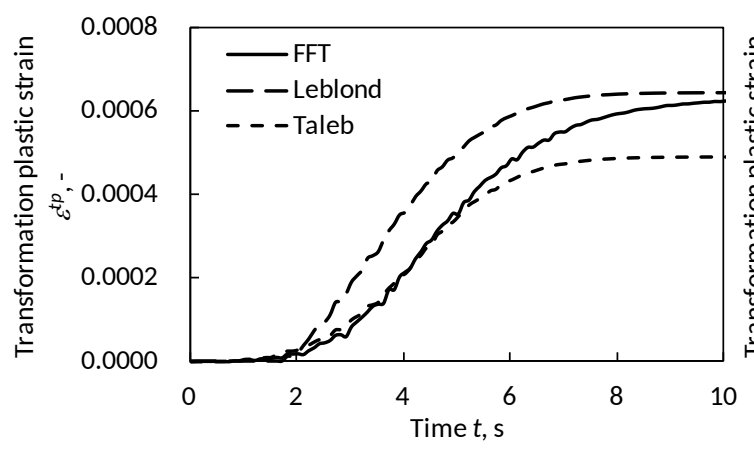

(a) $30 \mathrm{MPa}$

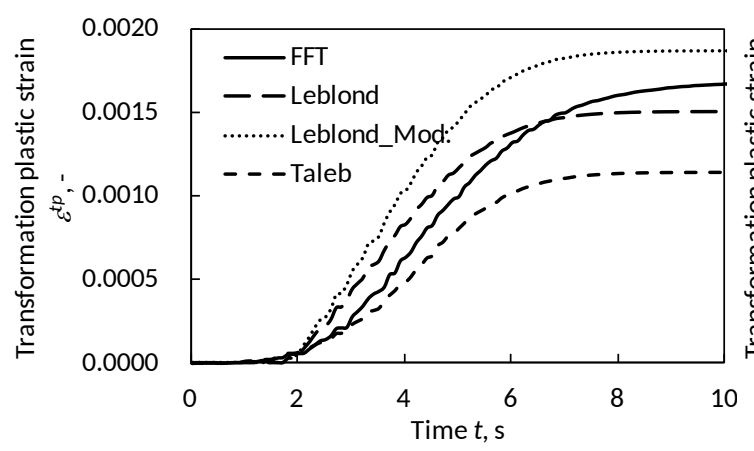

(c) $70 \mathrm{MPa}$

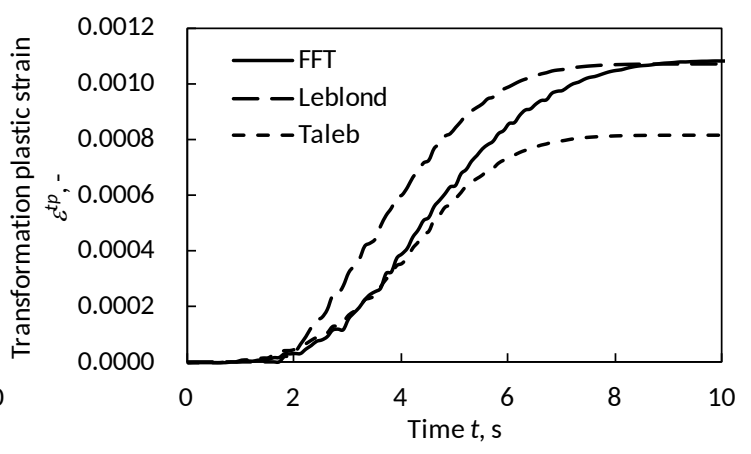

(b) $50 \mathrm{MPa}$

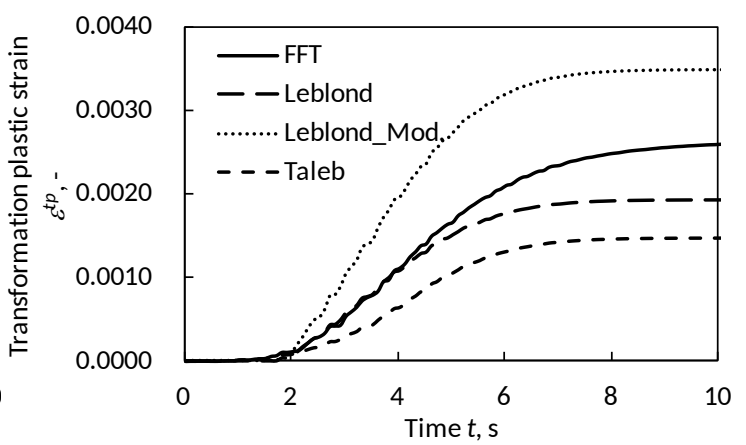

(d) $90 \mathrm{MPa}$

Fig. 13 Comparison of evolution of transformation plastic strain.

Next discussion is on the kinematic evolution of strain during phase transformation under applied stress. Fig. 13 shows the comparison of the evolution curves of the transformation plastic strain predicted by FFT, Leblond's model (33) and Taleb model (38). According to Fig. 13, Taleb's model and FFT results agree at the beginning of phase transformation process. By contrast, Leblond's model overestimates the transformation plastic strain at this stage especially under small applied stresses. This agreement between Taleb's model and FFT results at the beginning of transformation can be explained by the Taleb's modification which considers that the parent phase is not entirely elastoplastic at the beginning of phase transformation. The modification is rather important when transformation expansion is relatively small. Both Leblond and Taleb's models underestimate the transformation plastic strain during the second half of phase transformation. This seems to be caused by plastic deformation of the daughter phase which is neglected by the analytical models. Thus, it is counterbalanced by an underestimation of the transformation plastic strain during the final stage for Leblond's model. On the other hand, for Taleb's model, it underestimates whole transformation plastic strain because of the underestimation at the final stage (and due to precise estimation at its initial stage) caused by the inappropriate assumption of elastic daughter phase as seen below. 


\section{Effect of plastic deformation in daughter phase}

To enlighten the each contribution to transformation plasticity by parent and daughter phases, equivalent plastic strain of each phase is separately shown in Fig. 14.

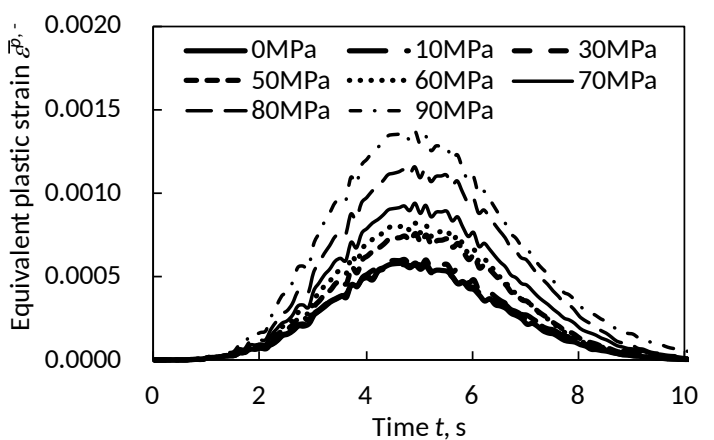

(a) parent phase

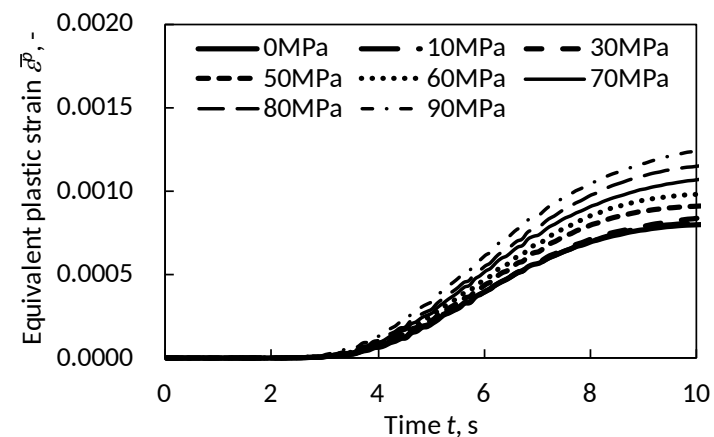

(b) daughter phase

Fig. 14 Strain evolutions of parent and daughter phases during phase transformation.

The equivalent plastic strain in parent phase takes maximum value when approximately $50 \%$ transformed. After $50 \%$, the equivalent plastic strain in parent phase decreases due to the decrease in volume fraction. The non-negligible strain in daughter phase accumulates in conjunction with the strain decrease in parent phase. Therefore, neglect of the strain in daughter phase is obviously a major agent for the underestimation at the end of the phase transformation.

These discussions lead a conclusion that the underestimation of Leblond's and Taleb's models in the latter half of phase transformation derives from the assumption of elastic daughter phase. To confirm this analysis, FFT numerical simulations with elastic daughter phase (infinite yield stress) are carried out. The comparison among FFT, Leblond's equation (33) and Taleb's equation (38) under several applied stresses is shown in Fig. 15.

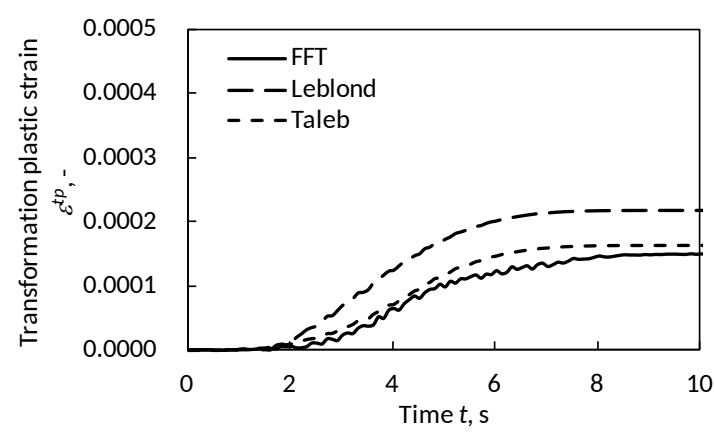

(a) $10 \mathrm{MPa}$

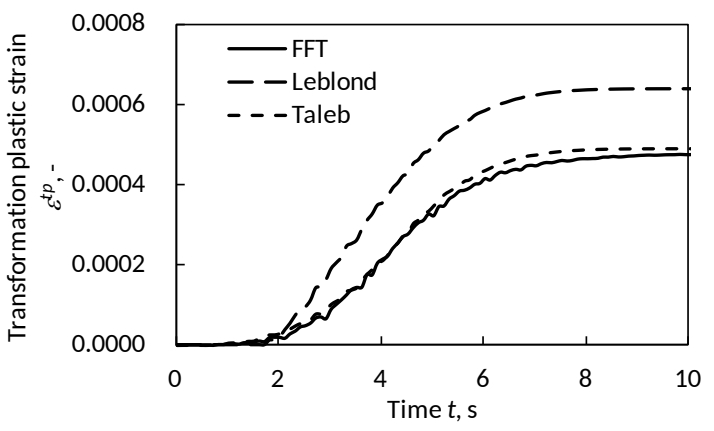

(b) $30 \mathrm{MPa}$ 


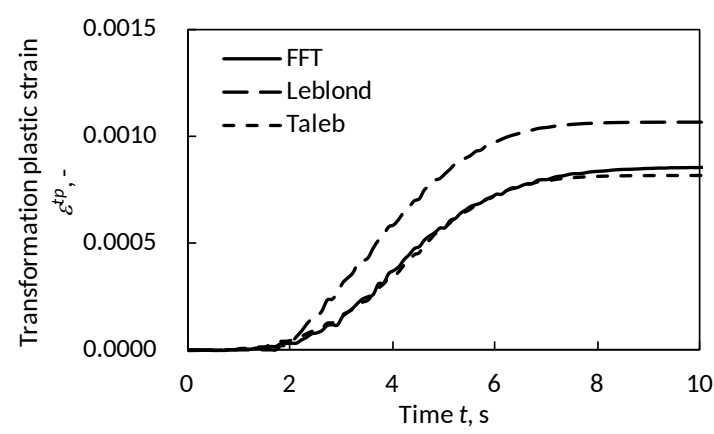

(c) $50 \mathrm{MPa}$

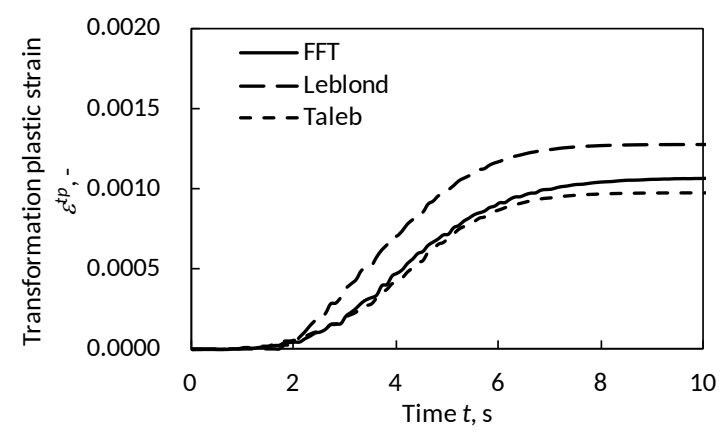

(d) $60 \mathrm{MPa}$

Fig. 15

Comparison of evolution of transformation plastic strain (daughter: elastic case).

Fig. 15 shows that Taleb's equation (38) closely agrees with FFT calculation results. Leblond's model overestimates the transformation plasticity, while Taleb's model agrees well with FFT during the whole transformation process when the daughter phase is elastic.

Fig. 16 represents the relation between applied stress and transformation plastic strain for daughter phase elastic condition. The transformation plasticity coefficient is $K_{P}=1.68 \times 10^{-5}\left(\mathrm{MPa}^{-1}\right)$ and again there is no significant difference between tensile and compressive stress conditions. Comparing to the daughter phase elastoplastic case, the transformation plasticity coefficient value is about $20 \%$ smaller for daughter phase elastic case. This appears also to be in quantitative agreement with the discrepancy in total transformation plastic strain between FFT numerical model and Taleb's model. This proved that plasticity in daughter phase should be incorporated especially when the daughter phase is relatively soft as ferrite phase.

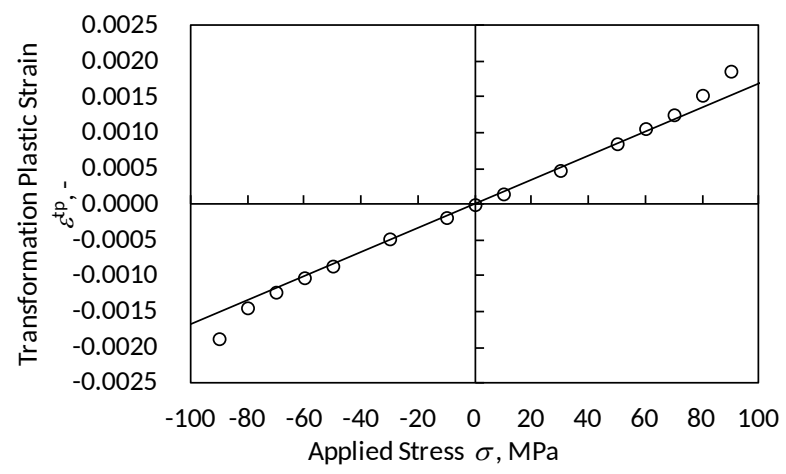

Fig. 16

Relation between applied stress and transformation plastic strain (pearlite: elastic case).

\section{Mechanism that causes nonlinearity under high applied stress}

When the applied stress is large, both Leblond's and Taleb's model underestimate transformation 
plastic strain as shown in Fig. 13 (c) and (d). In these cases, the linear relation between transformation plastic strain and applied stress is never satisfied (Fig. 6). The reason of this nonlinearity is investigated here by FFT computations.

Many models, such as Greenwood-Johnson [2] or Inoue [43], predict a linear relationship between applied stress and transformation plastic strain. Therefore, they are valid only for small applied stress conditions. As previously discussed, experimental results show that when the applied stress exceeds half the yield stress of the parent phase, nonlinearity occurs.

Leblond has analytically modelled transformation plasticity according to the Greenwood-Johnson mechanism [15]. This model contains 5 main hypotheses which are recalled here.

H1. The microscopic elastic compliance tensor may be equated to the macroscopic overall elastic compliance tensor.

H2. For small or moderately high applied stresses, the austenitic phase is entirely plastic, but the $\alpha$ phase remains elastic or its plastic strain rate remains always much smaller than that of the $\gamma$ phase.

H3. Both phases are ideal-plastic and obey the von Mises criterion and the Prandtl-Reuss flow rule.

H4. Correlations between $\delta \varepsilon_{1}^{e q} / \delta z$ and $s_{1}$ can be neglected.

H5. For small applied stresses, the average stress deviator in phase 1 is almost equal to the overall average stress deviator.

We now intend to check which assumptions remain valid, and which are not, for the high applied stress regime.

Hypothesis 1 is fulfilled because local elastic anisotropy does not significantly affect transformation plasticity.

Concerning hypothesis 2, Fig. 17 shows a probability density distribution of equivalent plastic strain when $50 \%$ transformed state calculated by FFT ( $64^{3}$ voxels, 100 grains). The results show that most voxels remain elastic, which is against the hypothesis. The larger the applied stress, the more voxels deform plastically. It means that even though there are many parts which remain elastic, this hypothesis cannot be the reason for the nonlinearity under high applied stress.
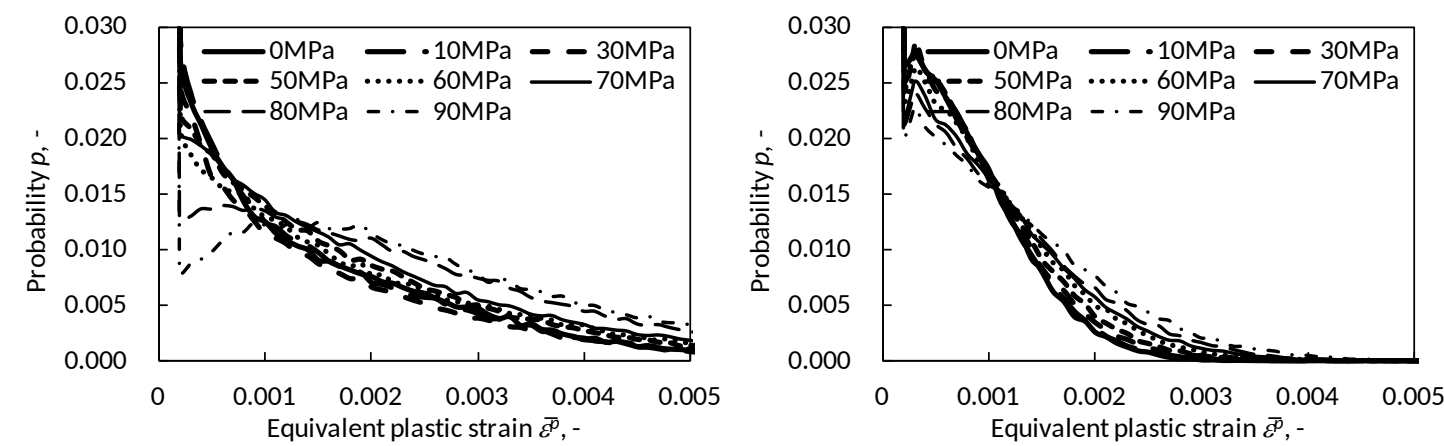

(a) Parent phase
(b) Daughter phase

Fig. 17 Equivalent plastic strain distribution at $\xi=0.5$.

We do not discuss hypothesis 3 since our calculations are not based on von Mises and Prandtl-Reuss models.

Hypothesis 4 can be verified by numerical results. The terms $\left\langle\delta \varepsilon_{1}^{e q} / \delta \xi \cdot s_{1}\right\rangle_{V_{1}}$ (Line 1) and $\left\langle\delta \varepsilon_{1}^{e q} / \delta \xi\right\rangle_{V_{1}} \cdot\left\langle s_{1}\right\rangle_{V_{1}}$ (Line 2) are calculated during phase transformation. The comparison of these two values is shown in Fig. 18.

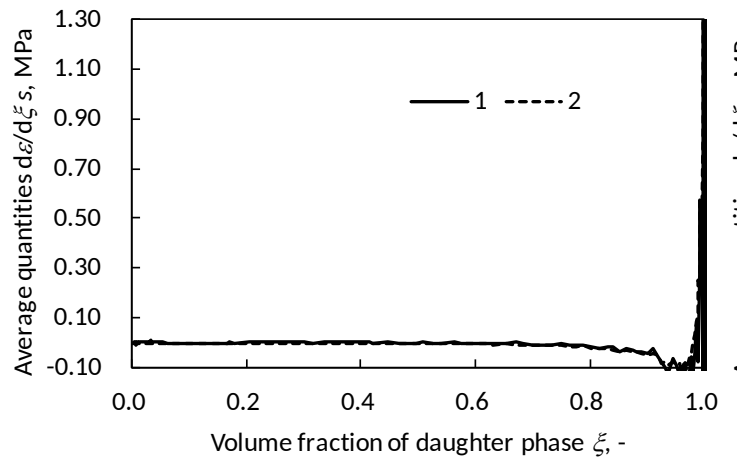

(a) $0 \mathrm{MPa}$

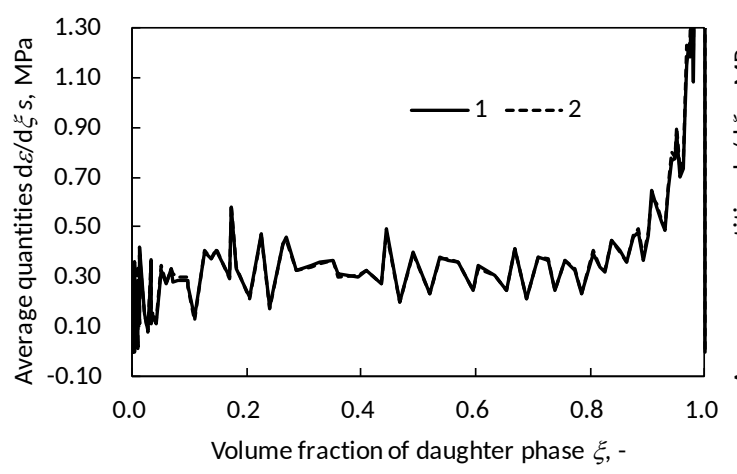

(c) $70 \mathrm{MPa}$

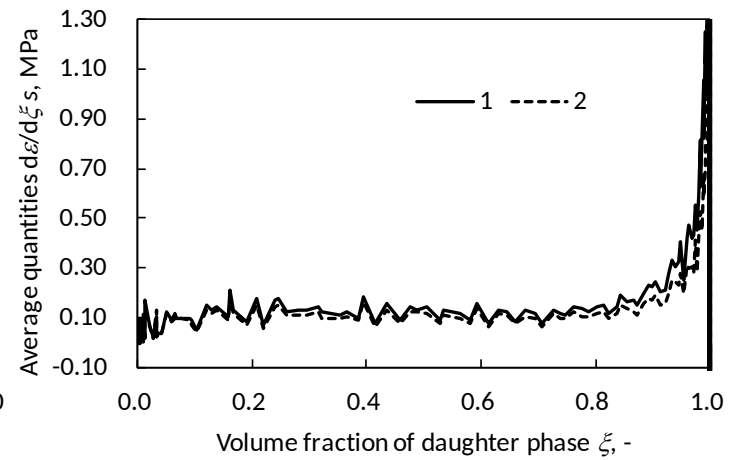

(b) $30 \mathrm{MPa}$

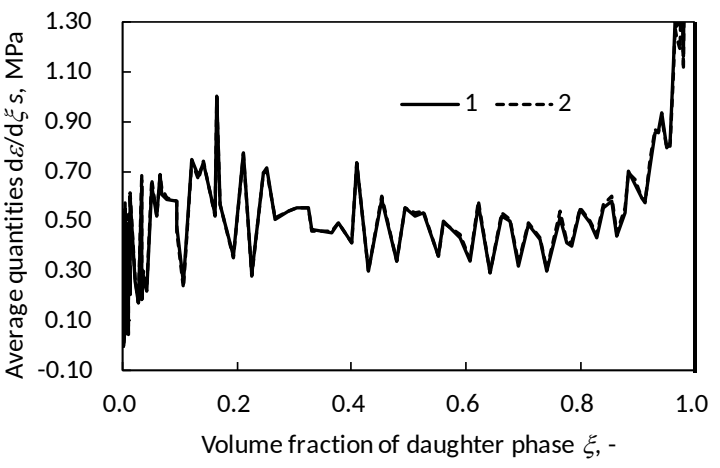

(d) $90 \mathrm{MPa}$

Fig. 18 Effect of correlation between $\delta \varepsilon_{1}^{e q} / \delta z(1)$ and $s_{1}(2)$ at several applied stress.

According to Fig. 18, it is clear that correlation between $\delta \varepsilon_{1}^{e q} / \delta z$ and $s_{1}$ can be neglected. Thus, the hypothesis 4 is well verified. In fact, as shown in Fig. 19, the resultant transformation plastic strains calculated with H.4 and without H.4 do not show substantive difference. This verification has also been performed by Leblond et al. [15] by using FEM with $\mathrm{J}_{2}$ plasticity. 


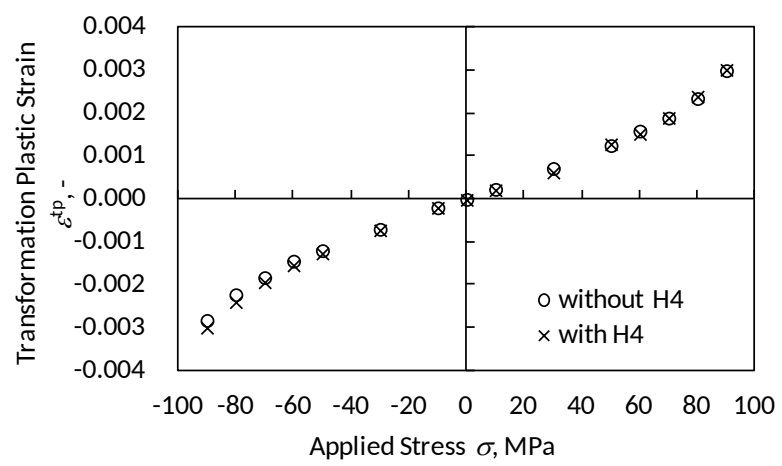

Fig. 19 Effect of correlation between $\delta \varepsilon_{1}^{e q} / \delta z$ and $s_{1}$.

Hypothesis 5 is now numerically verified. As for H.4, the average stress value of phase 1 and overall stress (average stress of both phase 1 and phase 2) are shown in Fig. 20.

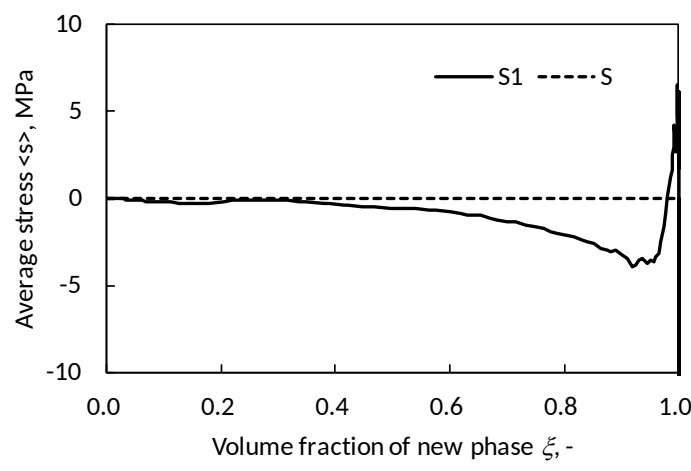

(a) $0 \mathrm{MPa}$

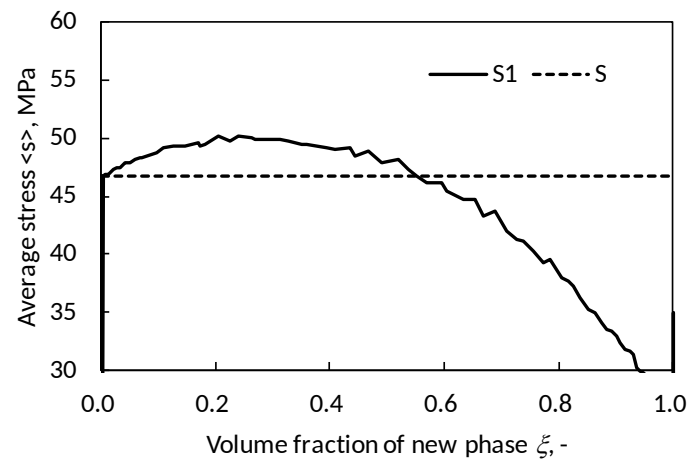

(c) $70 \mathrm{MPa}$

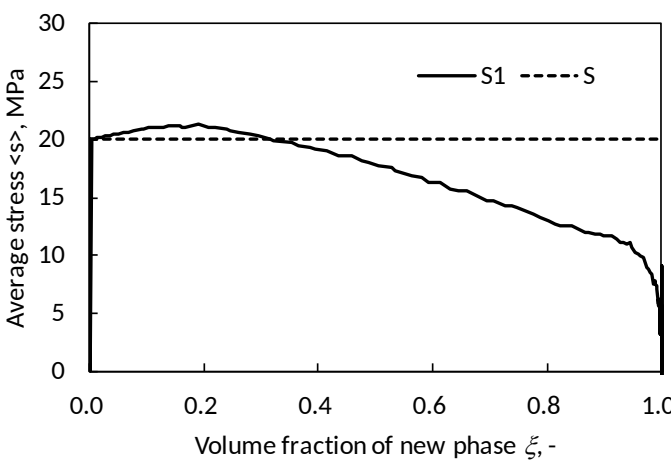

(b) $30 \mathrm{MPa}$

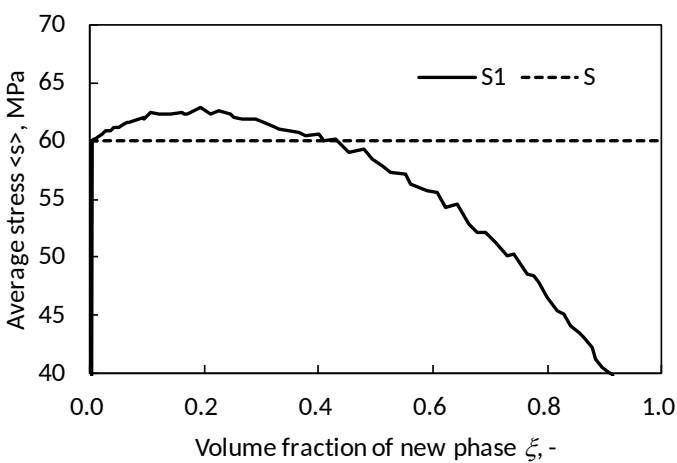

(d) $90 \mathrm{MPa}$

Fig. 20 Difference between average stress value of phase 1 and overall stress.

The maximum difference between average stress of phase 1 and overall stress until $50 \%$ phase transformation is less than $7 \%$. Hence, the hypothesis 5 is likely to be valid. To confirm this, the 
transformation plastic strain values with several applied stress are shown in Fig. $21 ;\left\langle\delta \varepsilon_{1}^{e q} / \delta z\right\rangle_{V_{1}}$. $\left\langle s_{1}\right\rangle_{V_{1}}$ without H.5 and $\left\langle\delta \varepsilon_{1}^{e q} / \delta z\right\rangle_{V_{1}} \cdot S$ with H.5. In this case, $\left\langle\delta \varepsilon_{1}^{e q} / \delta z\right\rangle_{V_{1}}$ is calculated by using analytical solution given by equation (32). As shown in Fig. 21, the hypothesis 5 is verified.

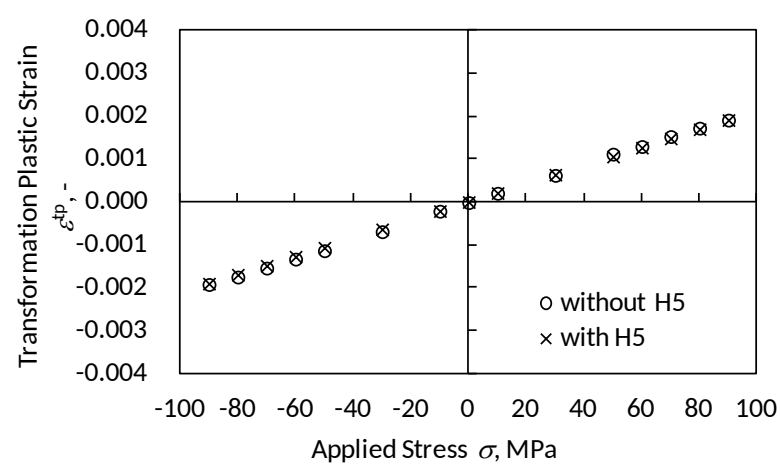

Fig. 21 Difference between average stress value of phase 1 and overall stress.

In contrast to Fig. 19, where one can observe the nonlinearity between applied stress and transformation plastic strain, Fig. 21 shows almost linear relation between them; the difference seems to arise from whether the term $\left\langle\delta \varepsilon_{1}^{e q} / \delta z\right\rangle_{V_{1}}$ is calculated by FFT or by analytic solution given by equation (32).

Fig. 22 shows the transformation plastic strains calculated by equation (31) in which the term $\left\langle\delta \varepsilon_{1}^{e q} / \delta z\right\rangle_{V_{1}}$.is given by analytical solution using equation (32) (1. circle symbol) and FFT solution (2. cross symbol). An important discrepancy is observed.

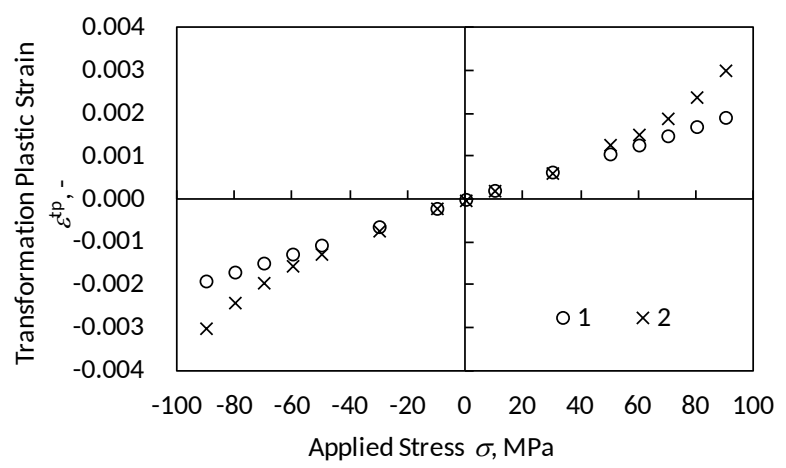

Fig. 22 Difference between analytical solution (circle) and FFT (cross) for $\left\langle\delta \varepsilon_{1}^{e q} / \delta \xi\right\rangle_{V_{1}}$. 
From the discussion above, it can be concluded that the estimation of term $\left\langle\delta \varepsilon_{1}^{e q} / \delta z\right\rangle_{V_{1}}$ is responsible for the nonlinearity under high applied stress condition. In addition, it shows that the analytical solution given by equation (32) is accurate for small applied stress but it is not valid under high applied stress value.

The reason why equation (32) is invalid under high applied stress is that the equivalent strain increment $\delta \varepsilon^{e q}(x)$ is supposed to be initiated only by transformation expansion. The assumption is valid under small applied stress but applied stress effect on equivalent strain increment is no longer negligible when applied stress is large.

\subsection{Application of Leblond's model for strain hardening materials [16] to pre-hardening case}

Finally, modified Leblond's for strain hardening materials (equation (35)) are compared with the FFT numerical results. In this calculation, back stress for $10 \%$ initial straining is estimated using equation (29). The obtained results for pre-tension case followed by stress-free transformation and $50 \mathrm{MPa}$ applied stress are shown in Fig. 23. The kinematic change in strain during phase transformation is surprisingly in good agreement between Leblond's model and numerical model especially for the stress free condition. The initial curves for $50 \mathrm{MPa}$ applied stress case are slightly different each other but the final strain values are very close.

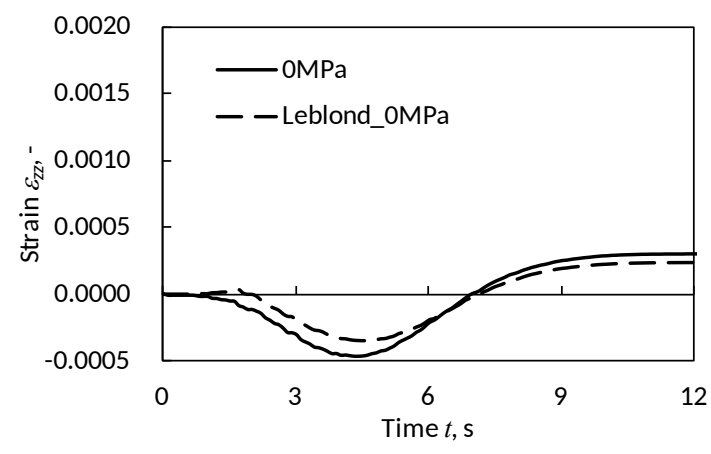

(a) $0 \mathrm{MPa}$

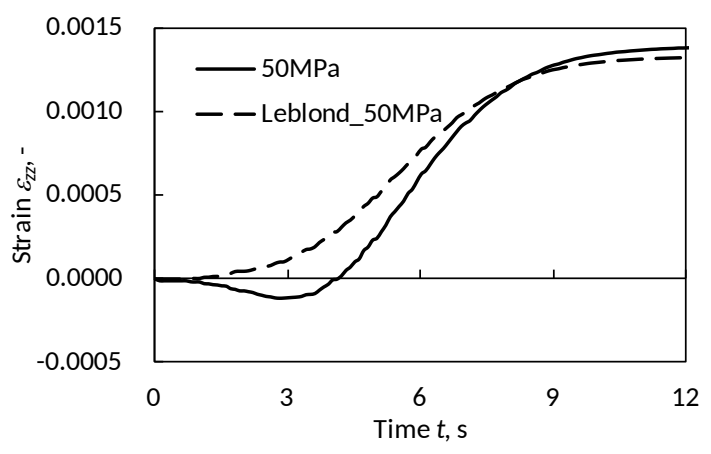

(b) $50 \mathrm{MPa}$

Fig. 23 Relation between FFT and Leblond model results of pre-tension case.

Another example is for pre-compression case. The back stress is again estimated by equation (29). The strain evolutions under stress absent and $50 \mathrm{MPa}$ applied stress cases are demonstrated in Fig. 24. The Leblond's analytical model for straining materials revealed that it is applicable to estimate transformation strain and transformation plasticity for both tensile and compressive pre-straining materials. 


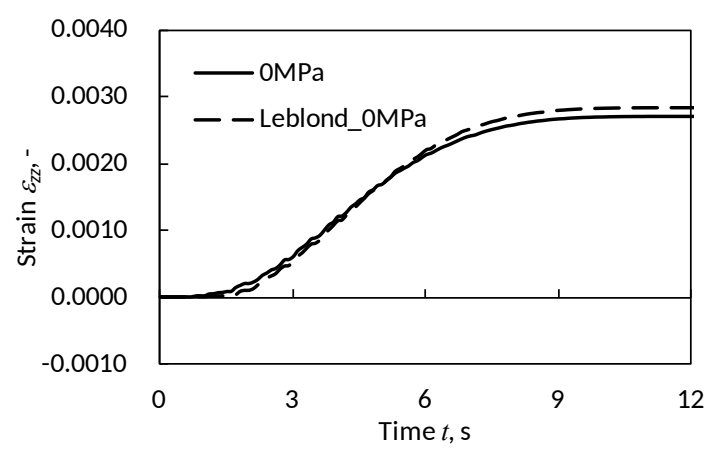

(a) $0 \mathrm{MPa}$

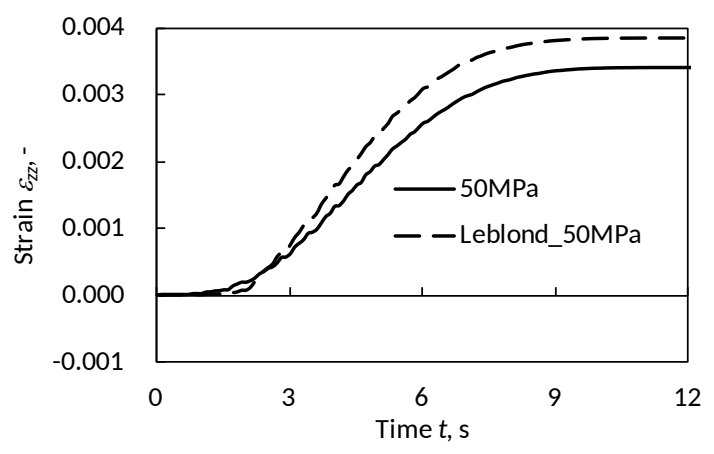

(b) $50 \mathrm{MPa}$

Fig. 24 Relation between FFT and Leblond model results of pre-compression case.

\section{Conclusion}

An elasto-plastic model for the prediction of the behaviour of a polycrystalline material subjected to diffusive phase transformation effect (transformation expansion) has been developed by using the discrete fast Fourier transform (FFT) numerical scheme. This alternative approach to the finite element method, based on Green functions, offers a simple framework to take into account the effect of the local volume expansion on the plastic response of the material. The model confirms that Greenwood-Johnson effect is one of the main mechanisms that cause transformation plasticity.

Then, the numerical model is applied to a more complicated phenomenon with pre-hardening effect. The pre-hardening leads to a distinct transformation strain evolution, namely pre-tension cause the small transformation dilatation (for the tensile direction) remaining total volume being same. The pre-compression case is totally opposite to the pre-tension case. This effect can be explained by the back stress (kinematic hardening of the austenite phase) and the anisotropy. The calculation results are in good agreement with experimental results by Taleb et al.

Finally, Leblond's and Taleb's models are compared with the FFT results shedding light on the quantitative analysis on the transformation plasticity. For the case of a small transformation expansion, the results by FFT are in quantitative agreement with the Leblond's model in terms of the final transformation plastic strain value. However, Leblond's model overestimates the transformation plastic strain at the beginning of phase transformation and underestimates it at the end. On the other hand, the Taleb's model shows a good agreement with the FFT numerical solution at the beginning of transformation but underestimates the transformation plastic strain during the latter half of phase transformation, which causes an underestimation of transformation plasticity. As Taleb has mentioned in his paper, the assumption that "the austenite phase is entirely plastic during phase transformation" is responsible for the overestimation of Leblond's model at the beginning of phase transformation. In addition, it is now clear that the underestimation of Leblond's model at the 
end of phase transformation can be explained by the plastic deformation in daughter phase. By using FFT results, it has been confirmed that the average ratio of the equivalent plastic strain increment over volume fraction increment, namely $\left\langle\delta \varepsilon_{1}^{e q} / \delta \xi\right\rangle_{V_{1}}$, is responsible for the nonlinearity.

For large transformation expansion case, both Leblond and Taleb models underestimate the transformation plastic strain. In the case of large transformation expansion, the period during which the parent phase is not entirely elastoplastic is short and thus the differences between models by Leblond and Taleb are small. Both models underestimate transformation plastic strain since they neglect the effect of an external stress on plastic strain of parent phase as well as the plastic strain in the daughter phase. These models are considered to be valid when the parent phase is much harder, i.e. bainitic or martensitic.

\section{References}

[1] C. L. Magee, "Transformation kinetics, Microplasticity and Aging of Martensite in Fe-31Ni," Ph.D. Thesis of Carnegie Institute of Technology, 1966.

[2] G. W. Greenwood , R. H. Johnson, "The Deformation of Metals under Small Stresses during Phase Transformations," Proc. Roy. Soc. 283A, pp. 403-422, 1965.

[3] F. D. Fischer, "Q-P. Sun and K. Tanaka, Transformation-induced plasticity(TRIP)," Appl. Mech. Rev., 49, 6, pp. 317-364, 1996.

[4] Y. Desalos, "Comportement dilatométrique et mécanique des materiaux d'austénite métastable d'un l'acier A533," IRSID, rapport no.95349401 MET44, pp. 28, 1981.

[5] E. Gautier, A. Simon, G. Beck, "Plasticité de transformation durant la transformation perlitique d'un acier eutectoïde," Acta Metall. Mater., 35, pp. 1367-1375, 1987.

[6] L. Taleb, N. Cavallo , F. Waeckel, "Experimental analysis of transformation plasticity," Int. J. Plasticity, 17, pp. 1-20, 2001.

[7] S. Petit-Grostabussiat, L. Taleb , J.-F. Jullien, "Experimental results on classical plasitcity of steels subjected to structural transformations,” Int. J. Plasticity, 20, pp. 1371-1386, 2004.

[8] H. -G. Lambers, S. Tschumak, H. J. Maier , D. Canadinc, "Pre-deformation-transformation plasticity relationship during martensitic transformation,” Mater. Sci. Eng. A527, pp. 625-633, 2010.

[9] A. Boudiaf, L. Taleb , M. A. Belouchrani, "Experimental analysis of the correlation between martensitic transformation plasticity and the austenitic grain size in steels," Eur. J. Mech. A. Solids, 30, pp. 326-335, 2011.

[10] M. J. Holzweissig, D. Canadinc , H. J. Maier, "In-situ characterization of transformation 
plasticity during an isothermal austenite-to-bainite phase transformation,” Mater. Charact., 65, pp. 100-108, 2012.

[11] T. Inoue, Z. G. Wang, K. Miyao, "Simulation of Quenching Process of Carburized Steel Gear Wheel under Metallo-Thermo-Mechanical CouplingElsevier Science Publishers, Paris, B. V. North-Holland,” Proc. IUTAM Thermomec. Coupling in Solids, pp. 257-262, 1987-5.

[12] D. Y. Ju, W. M. Zhang, Y. Zhang, "Modeling and experimental verification of martensitic transformation plastic behavior in carbon steel for quenching process," Mater. Sci. Eng., A 438440, pp. 246-260, 2006.

[13] J. Montalvo-Urquizo, Q. Liu , A. Schmidt, "Simulation of quenching involved in induction hardening including mechanical effects," Comp. Mater. Sci., 79, pp. 639-649, 2013.

[14] J. B. Leblond, G. Mottet, D. C. Devaux, "A Theoretical and Numerical Approach to the Plastic Behaviour of Steels During Phase Transformations-I. Derivation of General Relations,” J. Mech. Phys. Solids, 34, 4, pp. 395-409, 1986.

[15] J. B. Leblond, J. Devaux , J. C. Devaux, "Mathematical modelling of transformation plasticity in steels -I: Case of deal-plastic phases,” Int. J. Plasticity, 5, pp. 551-572, 1989.

[16] J. B. Leblond, "Mathematicl Modelling of Transformation Plasticity in Steels II: Coupling with Strain Hardening Phenomena,” Int. J. Plasticity, pp. 573-591, 1989.

[17] L. Taleb , F. Sidoroff, "A micromechanical modeling of the Greenwood-Johnson mechanism in transformation induced plasticity,” Int. J. Plasticity, 19, pp. 1821-1842, 2003.

[18] J. M. Diani, H. Sabar , M. Berveiller, "Mircromechanical modelling of the transformation induced plasticity (TRIP) phenomenon in steels,” Int. J. Engng Sci., 33, pp. 1921-1934, 1995.

[19] M. Cherkaoui, M. Berveiller, X. Lemoine, "Couplings between plasticity and martensitic phase transformation: overall behavior of polycrystalline TRIP steels," Int. J. Plasticity, 16, pp. 1215$1241,2000$.

[20] M. Fischlschweiger, G. Cailletaud , T. Antretter, "A mean-field model for transformation induced plasticity including backstress effects for non-proportional loadings," Int. J. Plasticity, 37, pp. 53-71, 2012.

[21] J. F. Ganghoffer, S. Denis, E. Gautier , A. Simon, "Finite element calculation of the micromecanics of a diffusional transformation,” Eur. J. Mech. A. Solids, 12, 1, pp. 21-32, 1993.

[22] F. Barbe, R. Quey, L. Taleb, "Numerical modelling of the plasticity induced during diffusive transformation. Case of a cubic array of nuclei,” Eur. J. Mech. A. Solids, 26, pp. 611-625, 2007.

[23] F. Barbe, R. Quey, L. Taleb , d. C. Souza, "Numerical modelling of the plasticity induced during diffusive transformation. An ensemble averaging approach for the case of random arrays of nuclei,” Eur. J. Mech. A. Solids, 27, pp. 1121-1139, 2008.

[24] F. Barbe , R. Quey, "A numerical modelling of 3D polycrystal-to-polycarystal diffusive phase 
transformations involving crystal plasticity,” Int. J. Plasticity, 27, pp. 823-840, 2011.

[25] H. Moulinec , P. Suquet, "A numerical method for computing the overall response of nonlinear composites with complex microstructure,” Comput. Method. Appl. M., 157, pp. 68-94, 1998.

[26] R. A. Lebensohn, R. Brenner, O. Castelnau , A. D. Rollett, "Orientation image-based micromechanical modellilng of subgrain texture evolution in polycrystalline copper," Acta Mater., 56, pp. 3914-3926, 2008.

[27] R. Brenner, R. A. Lebensohn, O. Castelnau, "Elastic anisotropy and yield surface estimates of polycrystals,” Int. J. Solids Struct., 46, pp. 3018-3026, 2009.

[28] S. -B. Lee, R. A. Lebensohn, A. D. Rollett, "Modeling the viscoplastic micromechanical response of two-phase materials using Fast Fourier Transfroms," Int. J. Plasticity, 27, pp. 707727, 2011.

[29] P. Suquet, H. Moulinec, O. Castelnau, M. Montagnat, N. Lahellec, F. Grennerat, P. Duval, R. Brenner, "Multi-scale modeling of the mechanical behavior of polycrystalline ice under transient creep," Procedia IUTAM, 3, pp. 64-78, 2012.

[30] R. A. Lebensohn, A. K. Kanjarla, P. Eisenlohr, "An elasto-viscoplastic formulation based on fast Fourier transforms for the prediction of micromechanical fields in polycrystalline materials,” Int. J. Plasticity, 32-33, pp. 59-69, 2012.

[31] P. Eisenlohr, M. Diehl, R. A. Lebensohn, F. Roters, "A spectral method solution to crystal elasto-viscoplasticity at finite strains," Int. J. Plasticity, 46, pp. 37-53, 2013.

[32] W. Ludwig, S. Schmidt, E. M. Lauridsene , H. F. Poulsen, "X-ray diffraction contrast tomography: a novel technique for three-dimensional grain mapping of polycrystals. i. direct beam case,” J. Appl. Cryst., 41, pp. 302-309, 2008.

[33] A. Belkhabbaz, R. Brenner, N. Rupin, B. Bacroix , J. Fonseca, "Prediction of the overall behavior of a 3D microstructure of austenitic steel by using FFT numerical scheme," Procedia Eng., 10, 1883-1888, 2011.

[34] F. Grennerat, M. Montagnat, O. Castenlau, P. Vacher, H. Moulinec, P. Suquet, P. Duval, "Experimental characterization of the intragranular strain field in columnar ice during during transient creep," Acta Mater., 60, pp. 3655-3666, 2012.

[35] J. W. Hutchinson, "Elastic-plastic behaviour of poly crystalline metals and composites," Proc. of Roy. Soc., A.319, pp. 247-272, 1970.

[36] D. Peirce, R. J. Asaro , A. Needleman, "An analysis of non-uniform and localized deformation in ductile single crystals," Acta Metall., 30, pp. 1087-1119, 1982.

[37] K. Arimoto, "Heat treatment simulations and their validation (in Japanese)," Arimotech Ltd. publishment, 2006.

[38] C. Cabus, "Etude et modélisation des textures de transformations de phases dans les aciers 
destinés à l'emboutissage," Doctoral thesis of University Paris 13, 2005.

[39] C. O. Frederick, P. J. Armstrong, "A mathematical representation of the multiaxial Bauschinger effect,” Mater. High Temp., 24, 1, pp. 1-26, 2007.

[40] T. Otsuka, Y. Wakasu, T. Inoue, "A simple identification of transformation plastic behaviour and some data for heat treating materials," Int. J. Mater. Prod. Technol., 24, 1-4, pp. 292-305, 2005.

[41] T. Otsuka, T. Akashi, S. Ogawa, T. Imai , A. Egami, "Effect of Volume Expansion on Transformation Plasticity during Ferrite and Martensite Transformation of Steel," J. Soc. Mater. Sci. JPN, 60, 10, pp. 937-942, 2011.

[42] G. C. Videau, G. Cailletaud, A. Pineau, "Experimental study of the transformation induced plasticity in a Cr-Ni-Mo-Al-Ti steel," J. Phys. IV, colloque C1, Supplèment au J de phisique III, 6, pp. 465-474, 1995.

[43] T. Inoue, "Unified Transformation-Thermoplasticity and the Application (in Japanese)," J. Soc. Mater. Sci. JPN., 56, 4, pp. 352-356, 2007. 
Appendix A

\section{Modified rate-independent model for elasto-plastic transient problems}

In this appendix, the computation of the plastic slip increments during a strain loading step is described. A modification of the Hutchinson's method (Hutchinson, 1970) is proposed for the elastoplastic transition. It allows to consider larger strain increment without affecting the overall response.

The plastic strain rate is given by summation of each slip rate on the slip systems, such that

$$
\dot{\varepsilon}_{i j}^{p}=\sum_{\alpha} \dot{\gamma}^{\alpha} p_{i j}^{\alpha}
$$

where, $\alpha$ is slip system $p_{i j}^{\alpha}$ is a Schemid tensor. So, the stress rate of elastoplastic problem can be found as:

$$
\dot{\sigma}_{i j}=C_{i j k l}\left(\dot{\varepsilon}_{i j}-\dot{\varepsilon}_{i j}^{p}\right)
$$

where, $\dot{\varepsilon}_{k l}$ is total strain rate and $C_{i j k l}(x)$ is elastic stiffness tensor. On the other hand, the constitutive equation of elastic problem is given simply as:

$$
\dot{\sigma}_{i j}=C_{i j k l} \dot{\varepsilon}_{i j}
$$

because total strain rate derives only from elastic strain.

When resolved shear stress $\tau^{\alpha}=\sigma_{i j} p_{i j}^{\alpha}$ exceeds the value $g^{\alpha}$, the material satisfies the yield condition and the problem becomes elastoplastic. The values $g^{\alpha}$ can be found according to the initial hardness of each slip system and their hardening law.

First of all, let us introduce Hutchinson method which allows one to find the magnitude of slip on each slip system. Suppose that the work hardening and resolved stress increment of $\alpha$ slip system during one step equates each other as depicted in Fig. A-1.

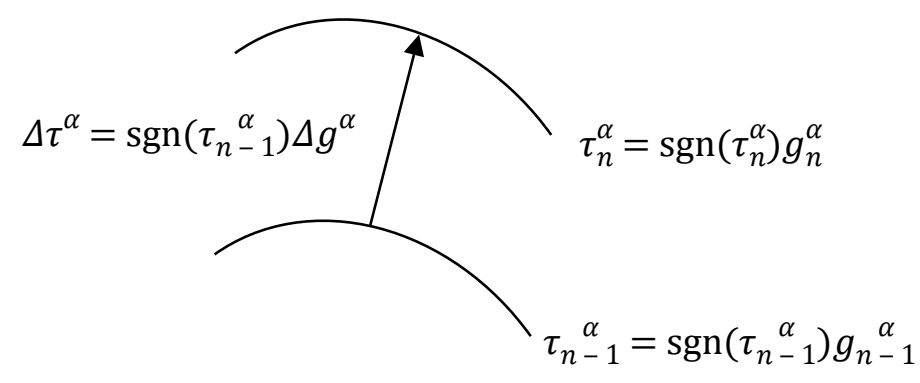

Fig. A-1 Work hardening and resolved stress increment during plastic deformation. 
It can also be expressed as following equations.

$$
\begin{aligned}
\Delta \tau^{\alpha} & =\operatorname{sgn}\left(\tau_{n-1}^{\alpha}\right) \Delta g^{\alpha} \\
\Delta \sigma_{i j} p_{i j}^{\alpha} & =\sum_{\beta} h^{\alpha \beta} \Delta \gamma^{\beta}
\end{aligned}
$$

When one introduces the matrix $X^{\alpha \beta}$, defined as:

$$
X^{\alpha \beta}=h^{\alpha \beta}+p_{i j}^{\alpha} C_{i j k l} p_{k l}^{\beta},
$$

the following relation can be obtained.

$$
\begin{aligned}
& \sum_{\beta} X^{\alpha \beta} \Delta \gamma^{\beta} \\
& =\sum_{\beta} h^{\alpha \beta} \Delta \gamma^{\beta}+\sum_{\beta} p_{i j}^{\alpha} C_{i j k l} p_{k l}^{\beta} \Delta \gamma^{\beta}=\Delta \sigma_{i j} p_{i j}^{\alpha}+p_{i j}^{\alpha} C_{i j k l} \varepsilon_{k l}^{p}=C_{i j k l}\left(\Delta \varepsilon_{k l}-\Delta \varepsilon_{k l}^{p}\right) p_{i j}^{\alpha}+p_{i j}^{\alpha} C_{i j k l} \\
& \varepsilon_{k l}^{p}=C_{i j k l} \Delta \varepsilon_{k l} p_{i j}^{\alpha}
\end{aligned}
$$

Thus, the slip increment is calculated by using matrix $Y^{\alpha \beta}$, the inverse matrix of $X^{\alpha \beta}$ :

$$
Y^{\alpha \beta}=\left(X^{\alpha \beta}\right)^{-1},
$$

such that;

$$
\Delta \gamma^{\alpha}=\sum_{\beta} Y^{\alpha \beta} C_{i j k l} \Delta \varepsilon_{k l} p_{i j}^{\beta}
$$

This result rigorously applies only for slip systems which are in the elastoplastic regime during the whole strain increment.

Second, let us consider the situation where the state of $\alpha$ slip system of previous increment was elastic and presently elastoplastic. Here, the trajectory of one step can be divided in two paths as depicted in Fig. A-2; one is elastic and the other is elastoplastic.

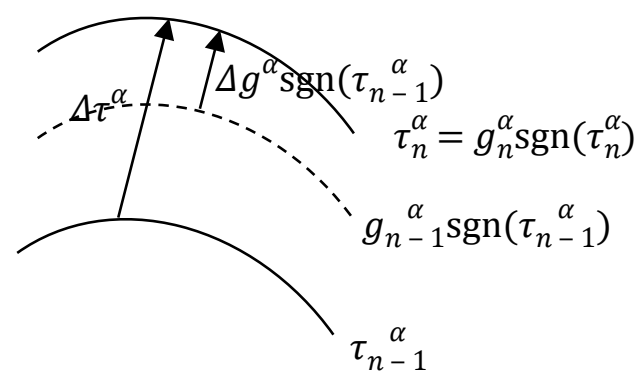

Fig. A-2 Division of trajectory in one step into two paths (elastic and elastoplastic). 
In this case, (A-3) can be rewritten as:

$$
\begin{aligned}
\tau_{n-1}^{\alpha}+\Delta \tau^{\alpha} & =\operatorname{sgn}\left(\tau_{n-1}^{\alpha}\right) g_{n-1}^{\alpha}+\operatorname{sgn}\left(\tau_{n}^{\alpha}\right) \Delta g^{\alpha} \\
\sigma_{i j} p_{i j}^{\alpha}+\Delta \sigma_{i j} p_{i j}^{\alpha} & =\operatorname{sgn}\left(\tau_{n-1}^{\alpha}\right) g_{n-1}^{\alpha}+\sum_{\beta} h^{\alpha \beta} \Delta \gamma^{\beta}
\end{aligned}
$$

And we obtain the final form of slip increment:

$$
\Delta \gamma^{\alpha}=\sum_{\beta} Y^{\alpha \beta}\left(\sigma_{i j} p_{i j}^{\beta}+C_{i j k l} \Delta \varepsilon_{k l} p_{i j}^{\beta}-\operatorname{sgn}\left(\tau_{n-1}^{\alpha}\right) g_{n-1}^{\alpha}\right)
$$

Uniaxial tensile numerical tests using the FFT numerical scheme with the two proposed method (Results A-7 and A-9)) have been carried out.

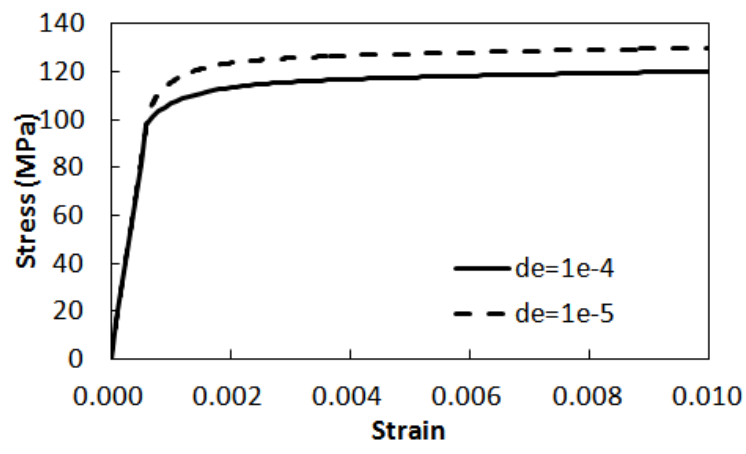

Fig. A-3 Calculated stress-strain curves with different strain increment par step.

Fig. A-3 shows calculated stress-strain curves with strain increment par step of $10^{-4}$ and $10^{-5}$. It is observed that a large strain increment leads to a softer response. This is due to the early plastic flow induced by the implicit method: plastic flow is assumed during the whole strain increment whereas the initial state is purely elastic.

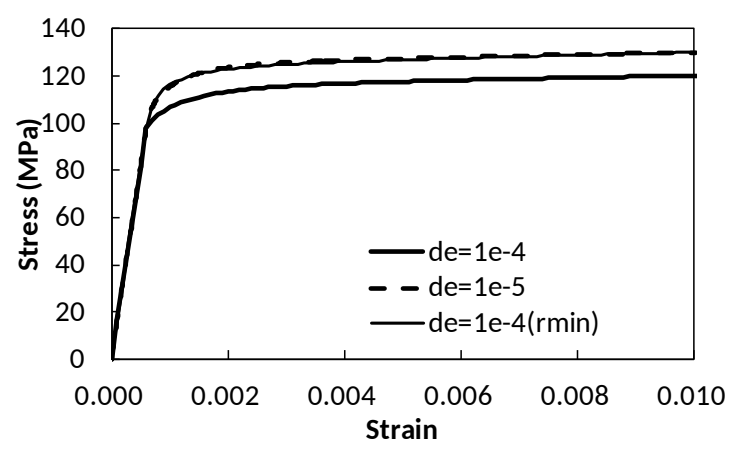

Fig. A-4 Calculated stress-strain curves with the two proposed methods for the determination 
of the plastic slip increments.

Fig. A-4 is a comparison among two different strain increments $\left(10^{-4}\right.$ and $\left.10^{-5}\right)$ in one step with using Fig. A-4 shows a comparison for two different strain increments $\left(10^{-4}\right.$ and $\left.10^{-5}\right)$. equation (A-7) by using equation (A-7) and the case of increment $10^{-4}$ using equation (A-9). The response with the new method agrees very well with the reference solution even though the strain increment is large. 


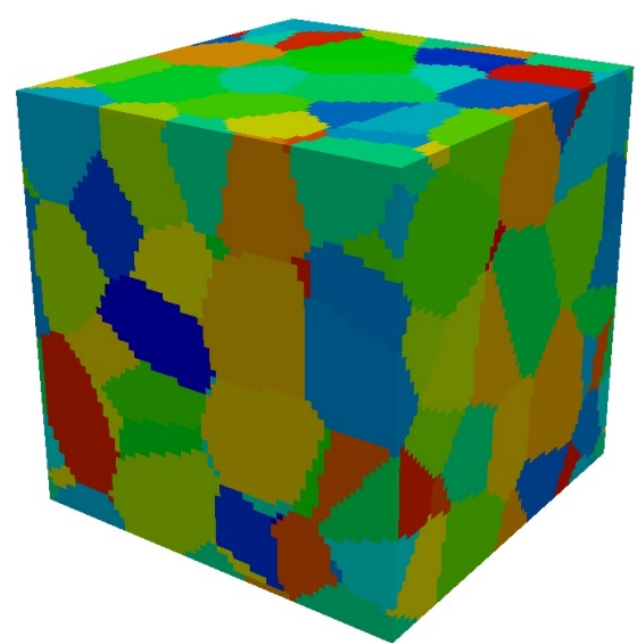

(a) Initial austenite phase

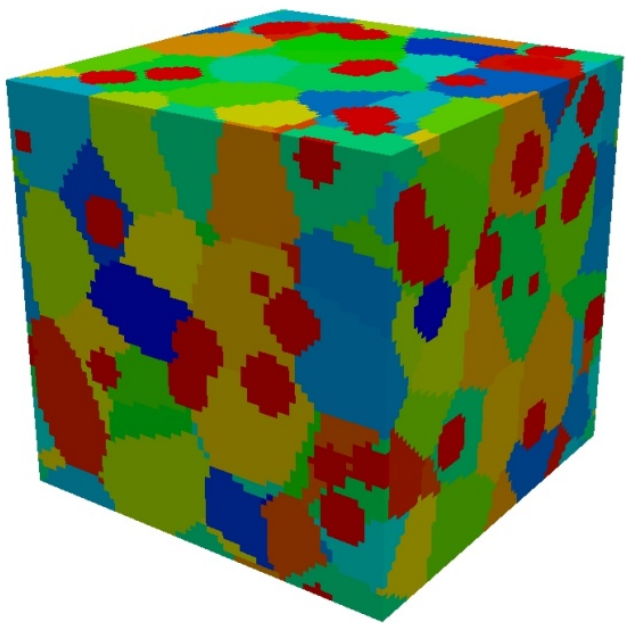

(b) During phase transformation

Fig. 1 Sample Voronoi tessellation and phase transformation; 100 grains in $64^{3}$ cubic space.

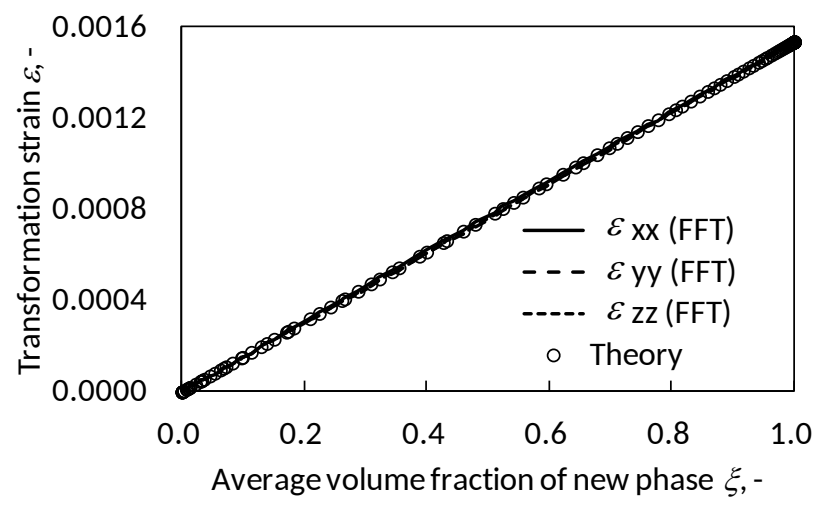

Evolution of the macroscopic strain with the volume fraction of transformed

Fig. 2 phase (no applied stress, isotropic polycrystal).

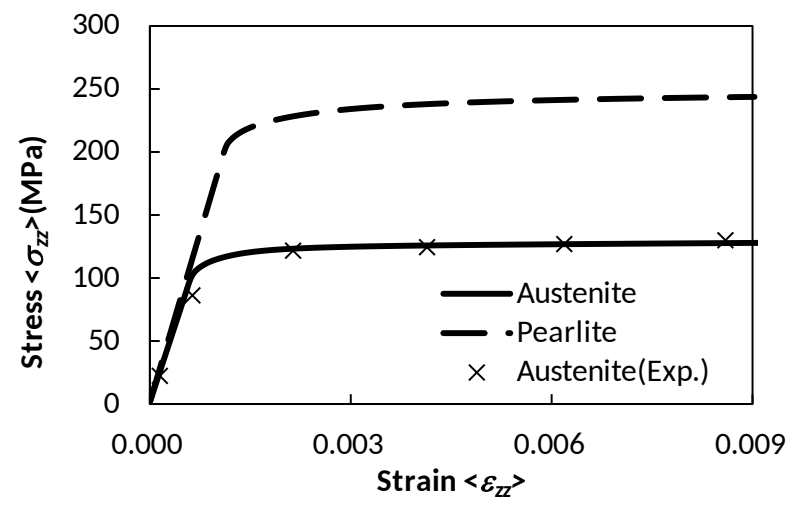

Fig. 3 Macroscopic stress/strain curves of Austenite and Pearlite phase 
(calculations and experiment at $670^{\circ} \mathrm{C}$ ).

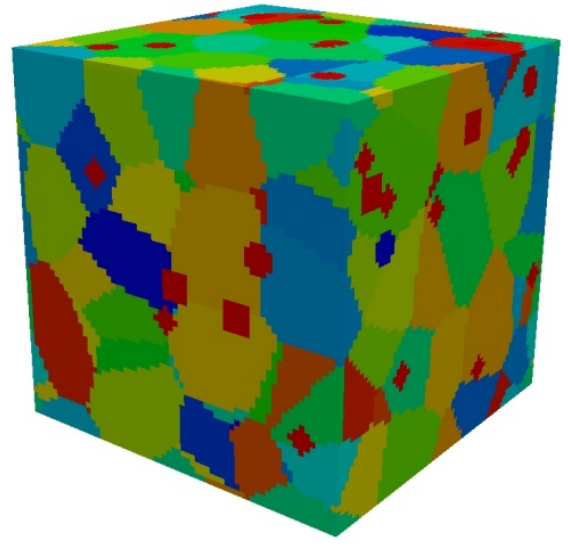

(a) phase distribution (3\%)

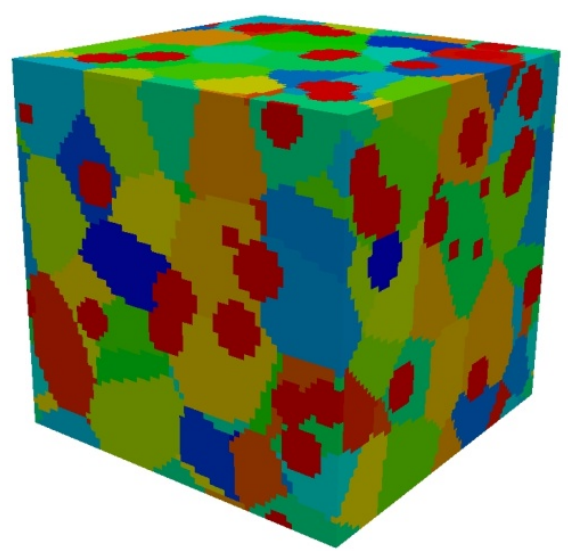

(c) phase distribution $(10 \%)$

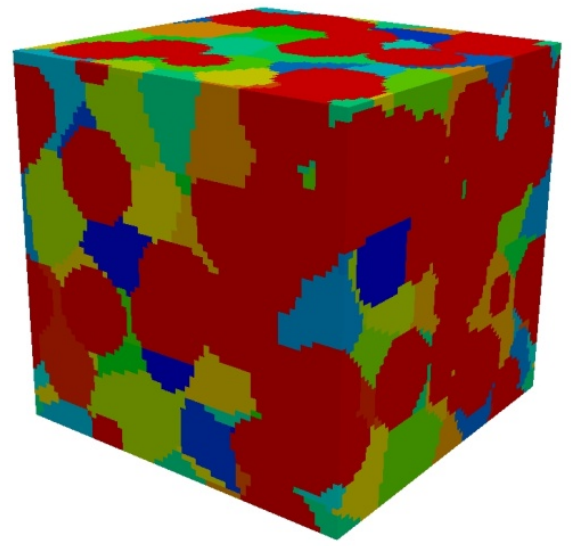

(e) phase distribution (50\%)

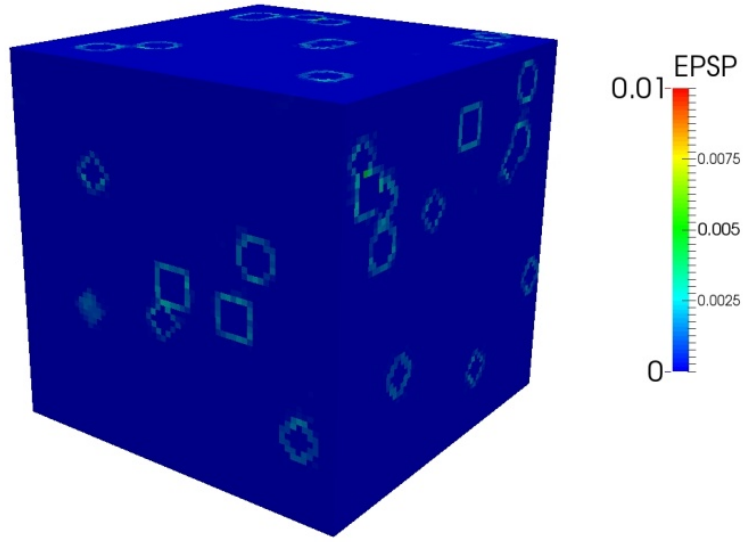

(b) equivalent plastic strain in mother phase (3\%)

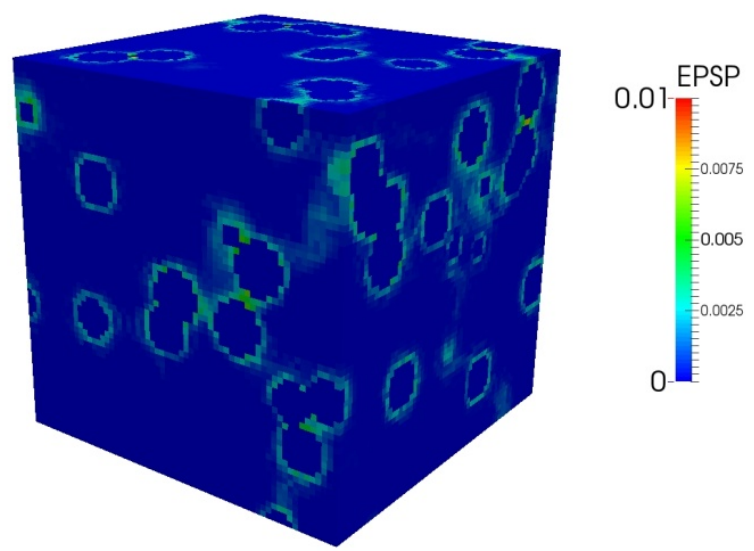

(d) equivalent plastic strain in mother phase (10\%)
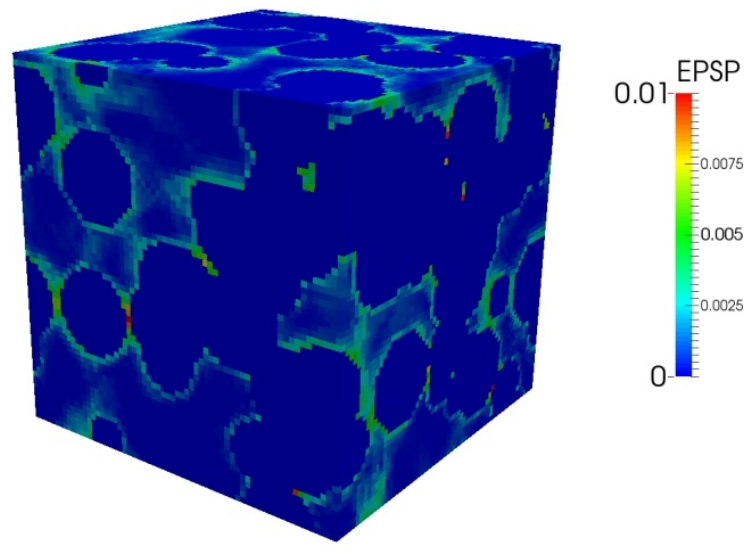

(f) equivalent plastic strain in mother phase (50\%)

Fig. 4 Evolution of volume fraction of new phase and macroscopic total strain. 


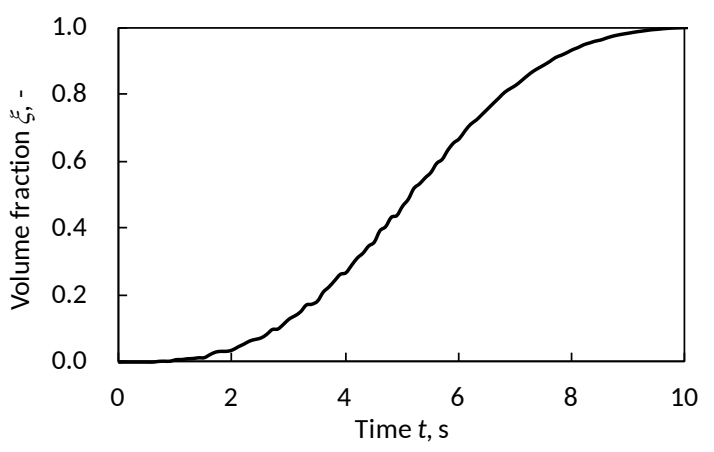

(a) Evolution of volume fraction

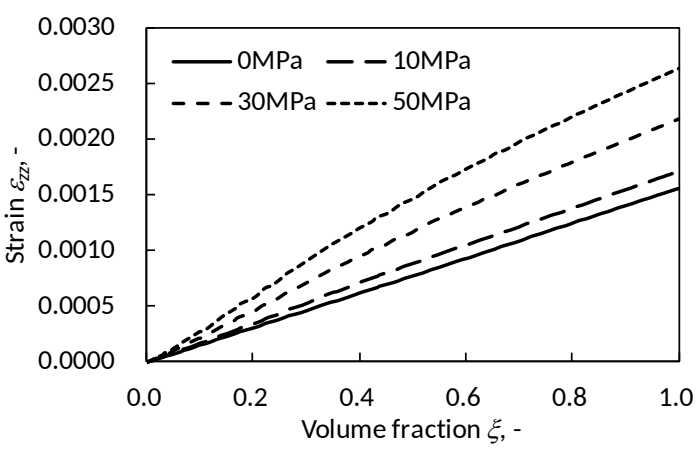

(b) Evolution of total strain

Fig. 5 Evolution of volume fraction of new phase and macroscopic total strain.

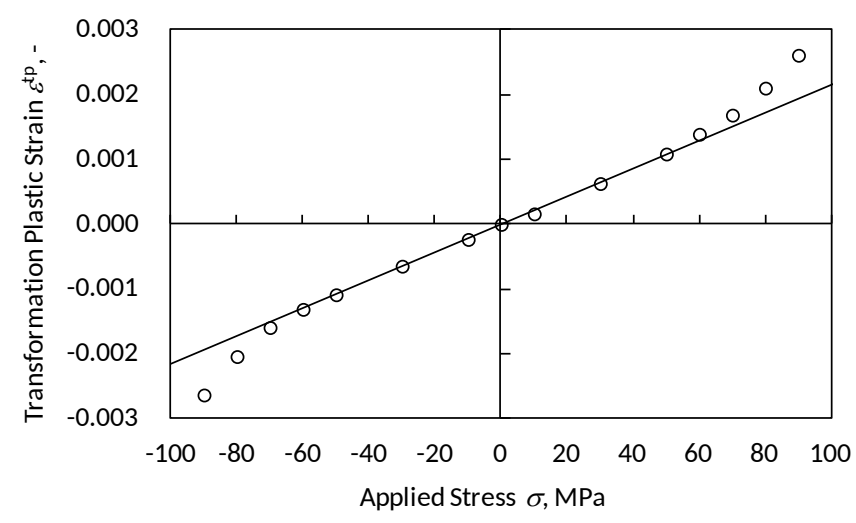

Fig. 6 Relation between applied stress and transformation plastic strain.

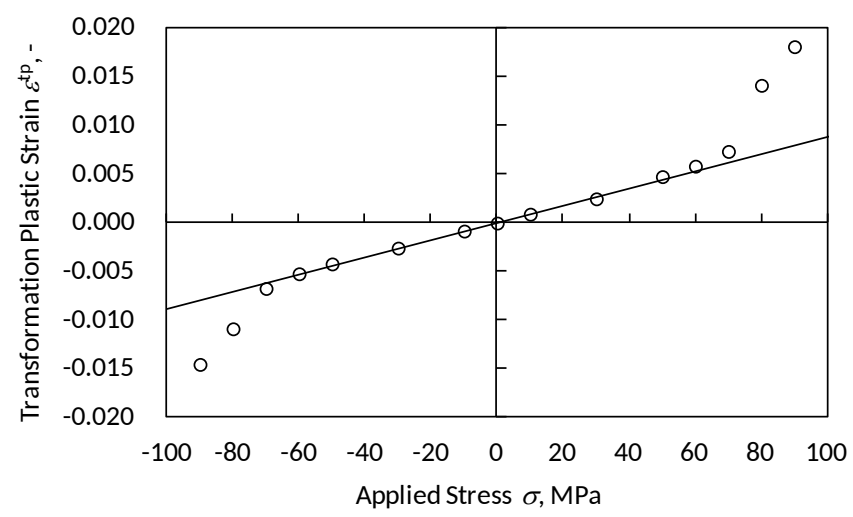

Fig. 7 Relation between applied stress and transformation plastic strain. 


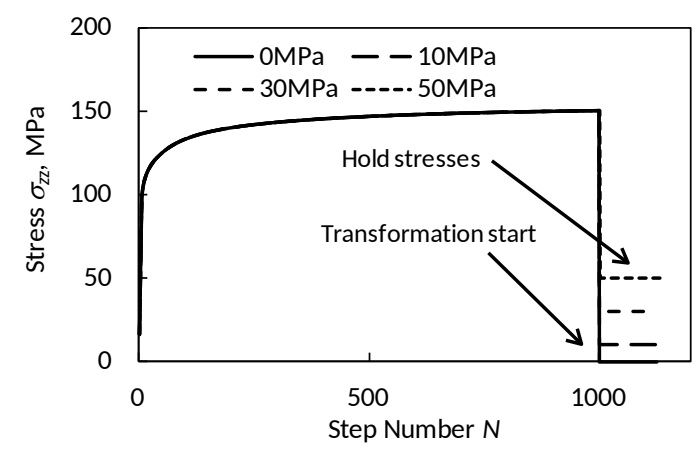

Fig. 8 Calculation sequence for pre-tension transformation plasticity.

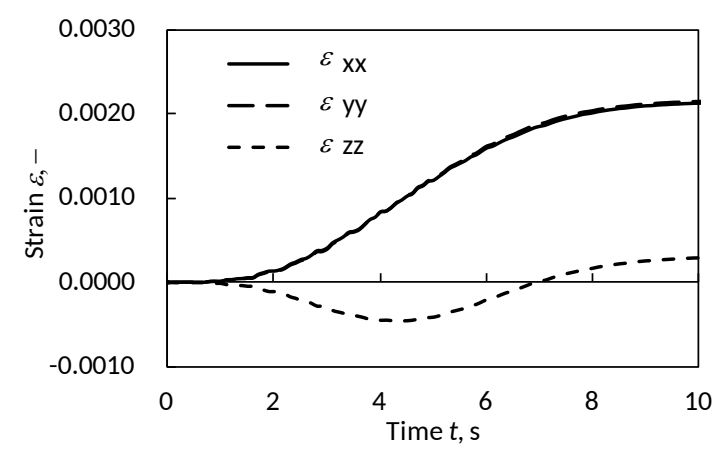

Fig. 9 Transformation strain with pre-tension.

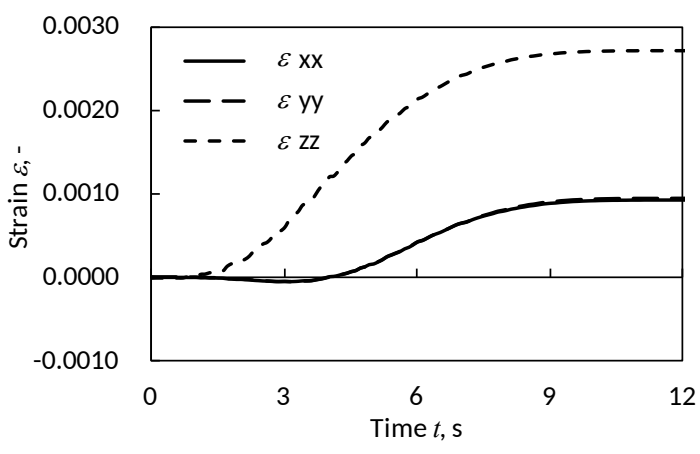

Fig. 10 Transformation strain with pre-compression. 


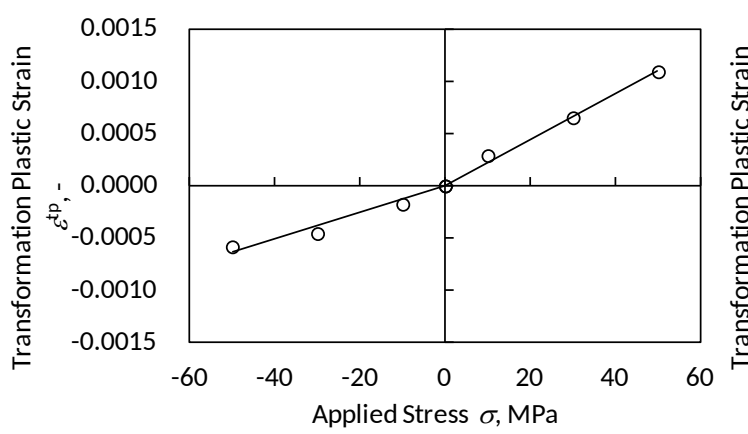

(a) pre-tension

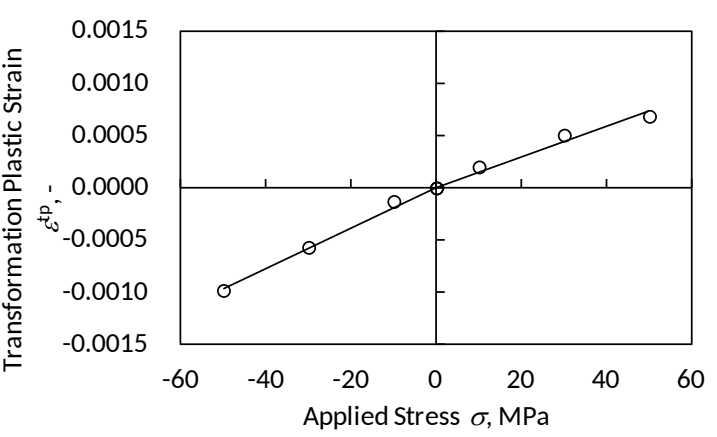

(b) pre-compression

Fig. 11 Relation between transformation plastic strain and applied stress.

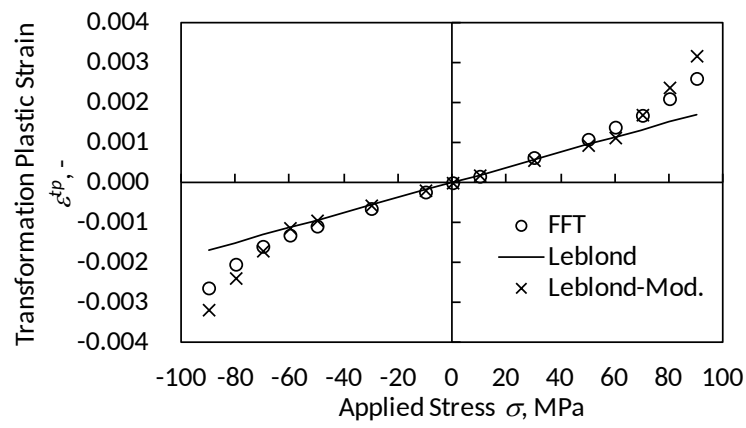

Fig. 12 Relation between applied stress and transformation plastic strain.

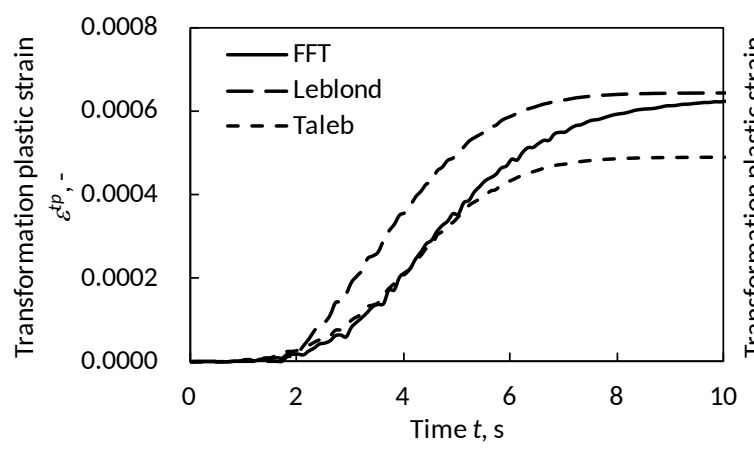

(a) $30 \mathrm{MPa}$

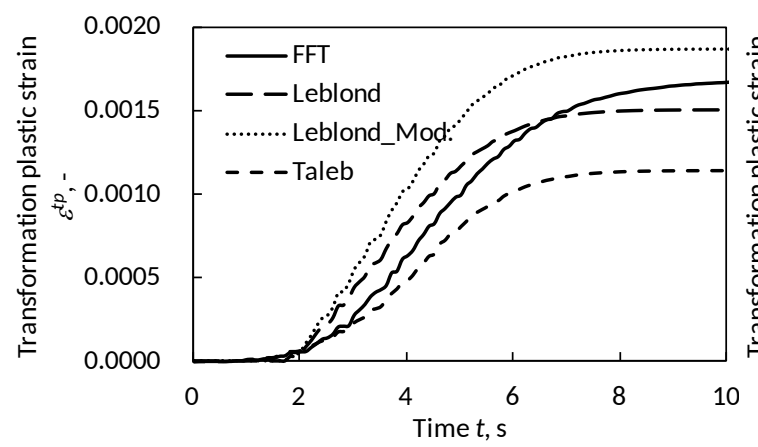

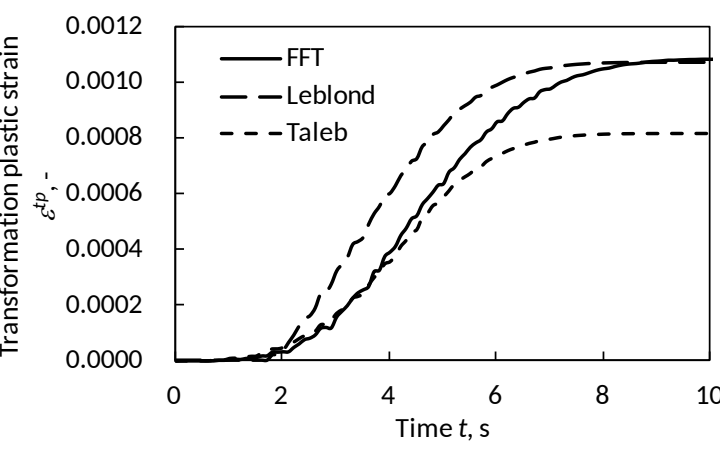

(b) $50 \mathrm{MPa}$

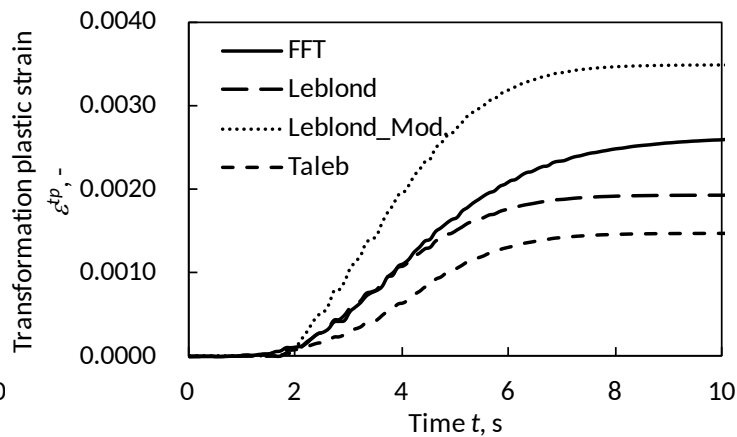



(c) $70 \mathrm{MPa}$
(d) $90 \mathrm{MPa}$

Fig. 13 Comparison of evolution of transformation plastic strain.

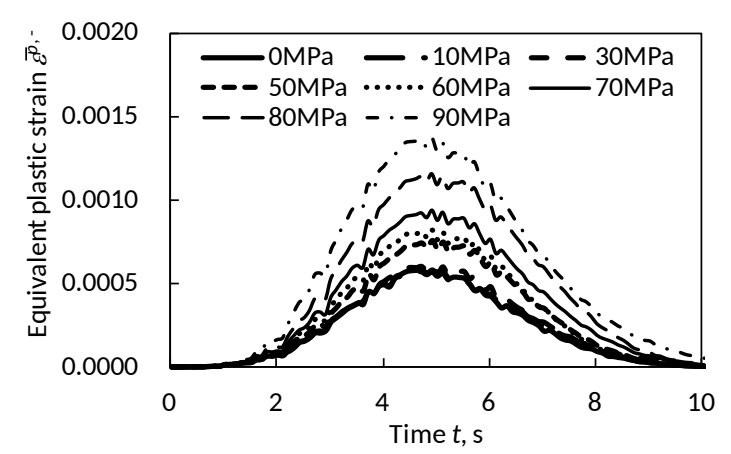

(a) parent phase

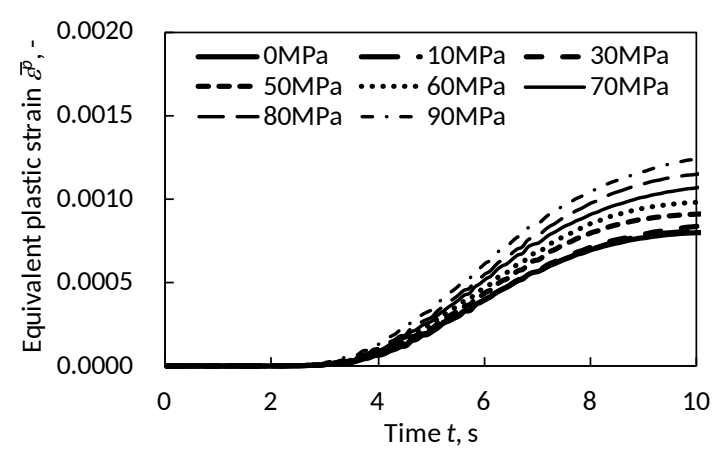

(b) daughter phase

Fig. 14 Strain evolutions of parent and daughter phases during phase transformation.

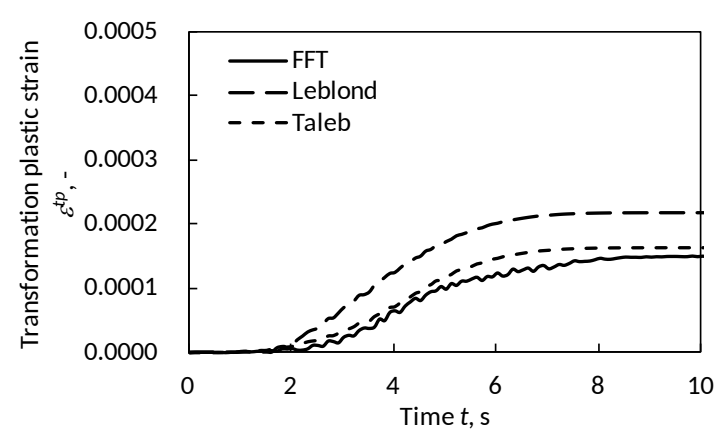

(a) $10 \mathrm{MPa}$

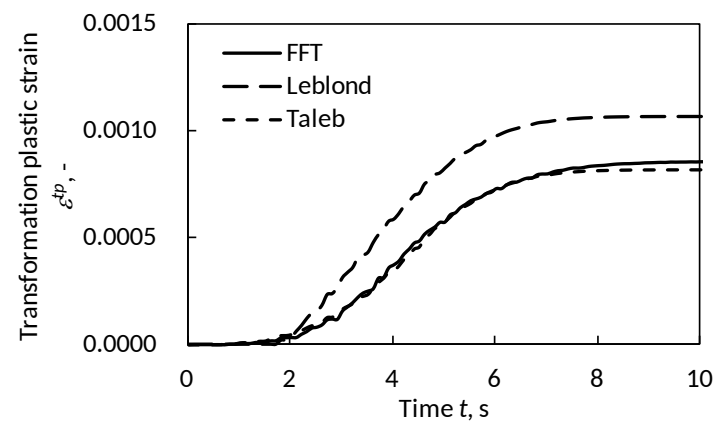

(c) $50 \mathrm{MPa}$

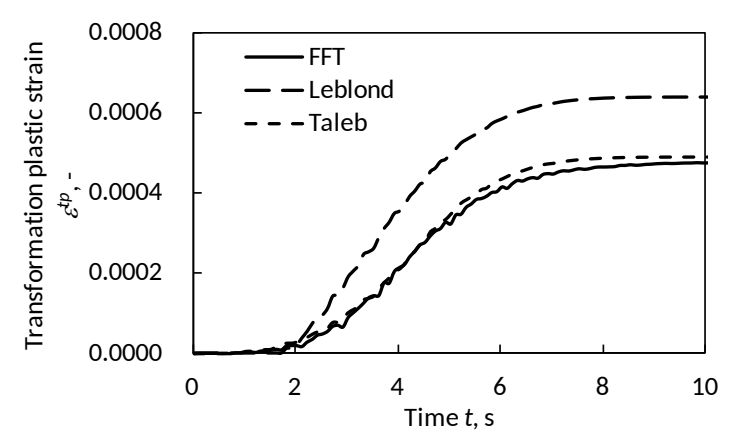

(b) $30 \mathrm{MPa}$

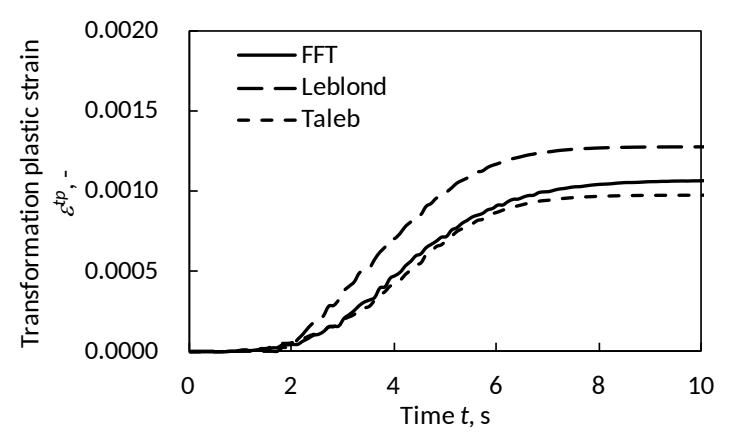

(d) $60 \mathrm{MPa}$

Fig. 15

Comparison of evolution of transformation plastic strain (daughter: elastic case). 


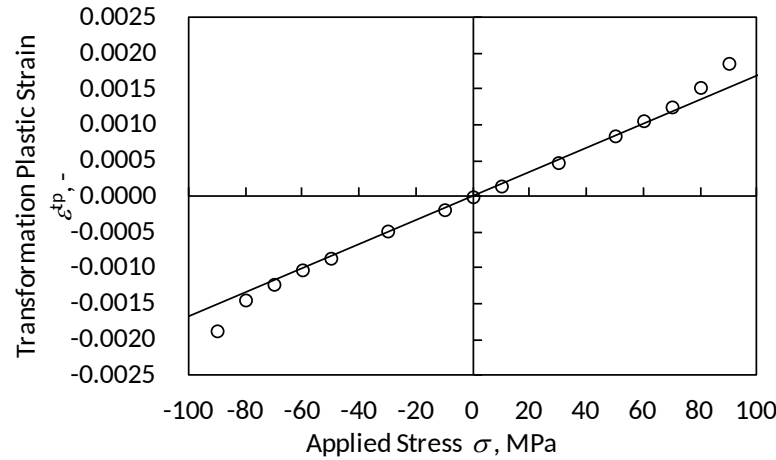

Fig. 16

Relation between applied stress and transformation plastic strain (pearlite: elastic case).

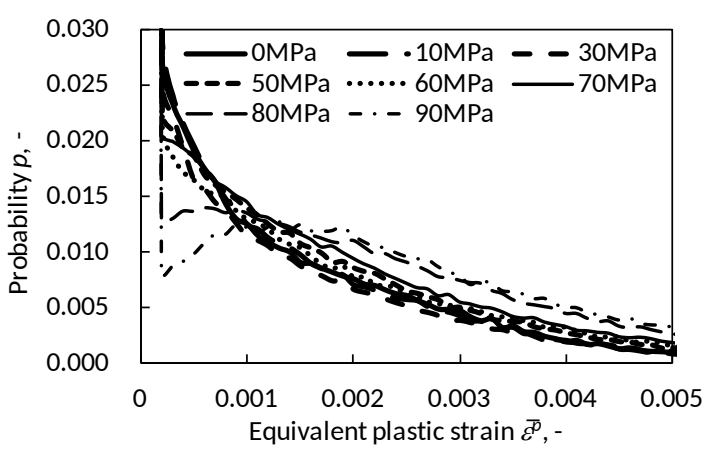

(a) Parent phase

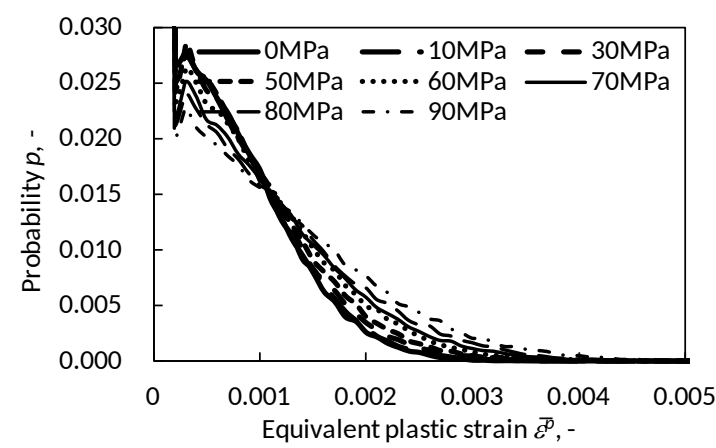

(b) Daughter phase

Fig. 17 Equivalent plastic strain distribution at $\xi=0.5$.

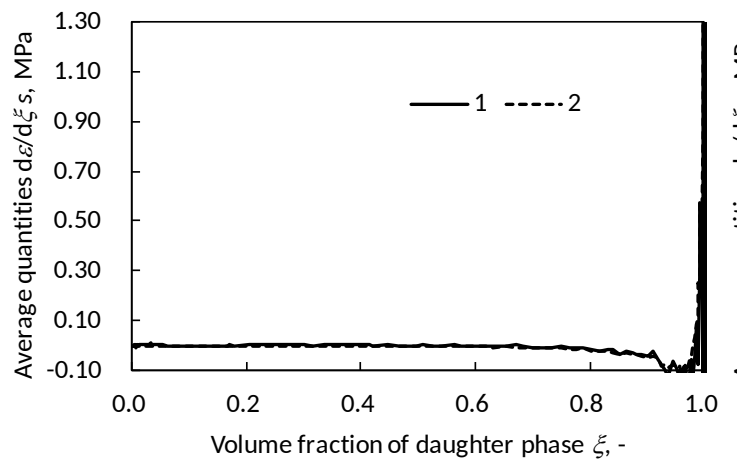

(a) $0 \mathrm{MPa}$

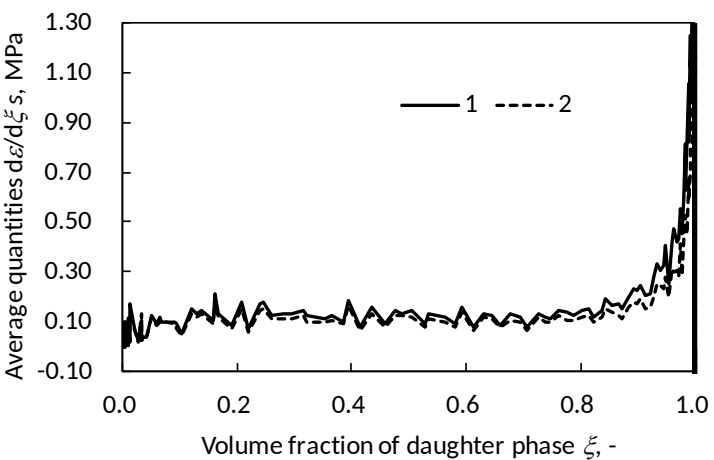

(b) $30 \mathrm{MPa}$ 


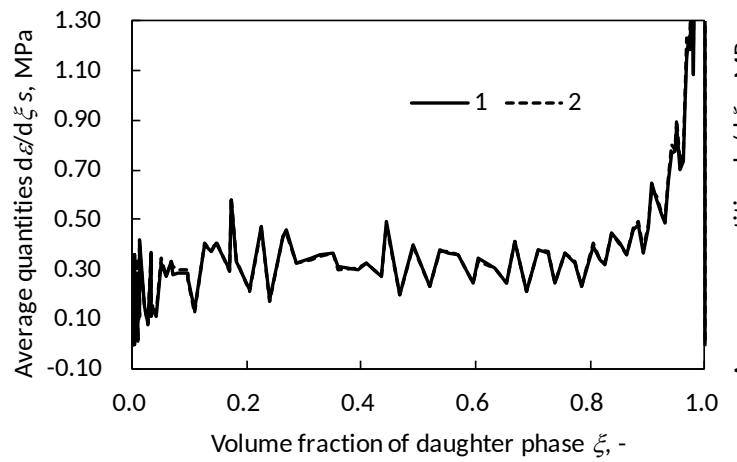

(c) $70 \mathrm{MPa}$

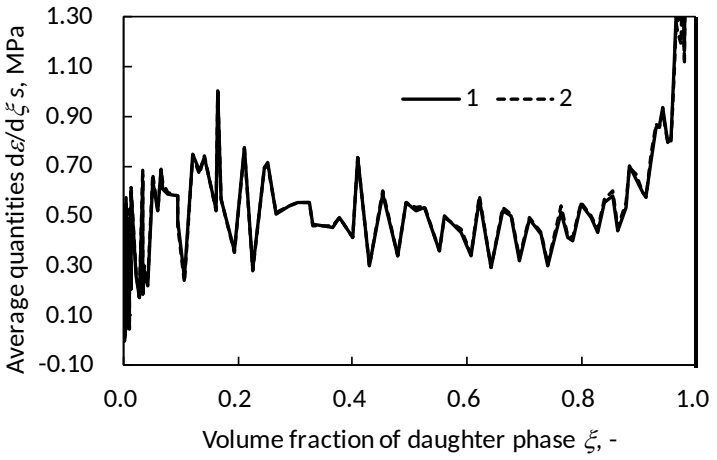

(d) $90 \mathrm{MPa}$

Fig. 18 Effect of correlation between $\delta \varepsilon_{1}^{e q} / \delta z(1)$ and $s_{1}(2)$ at several applied stress.

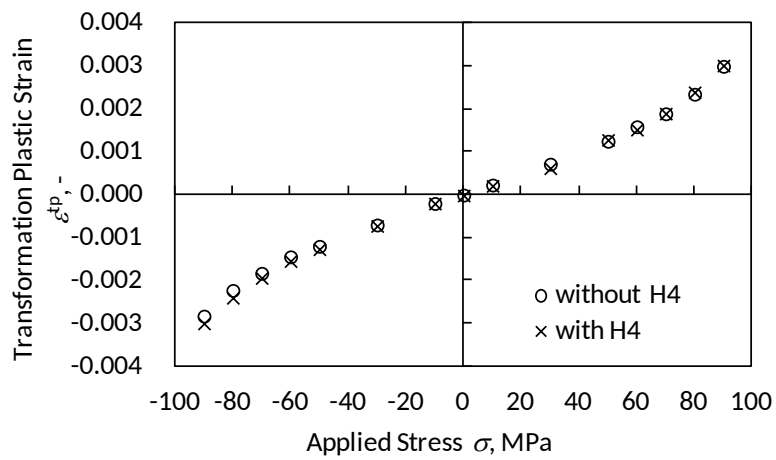

Fig. 19 Effect of correlation between $\delta \varepsilon_{1}^{e q} / \delta z$ and $s_{1}$.

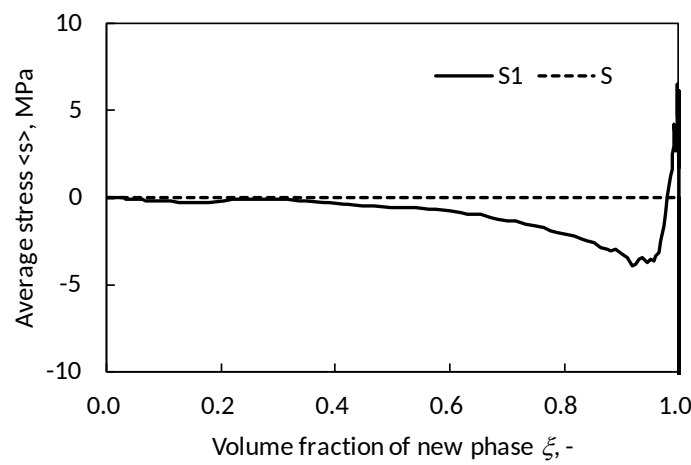

(a) $0 \mathrm{MPa}$

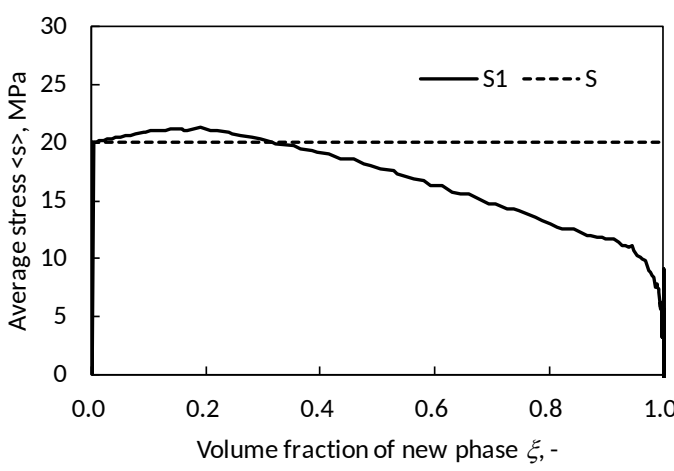

(b) $30 \mathrm{MPa}$ 


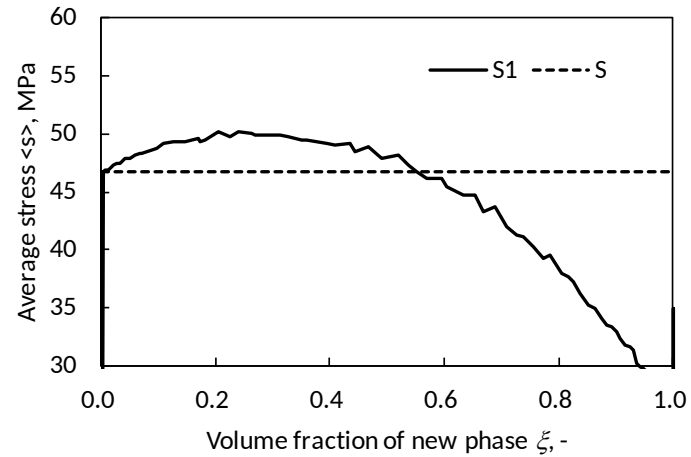

(c) $70 \mathrm{MPa}$

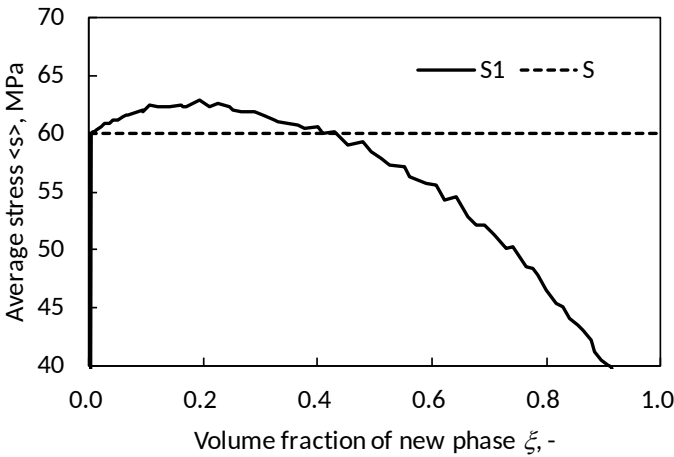

(d) $90 \mathrm{MPa}$

Fig. 20 Difference between average stress value of phase 1 and overall stress.

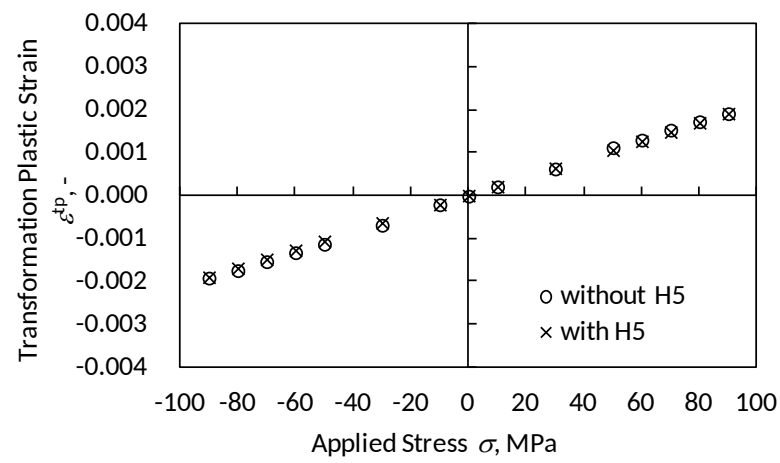

Fig. 21 Difference between average stress value of phase 1 and overall stress.

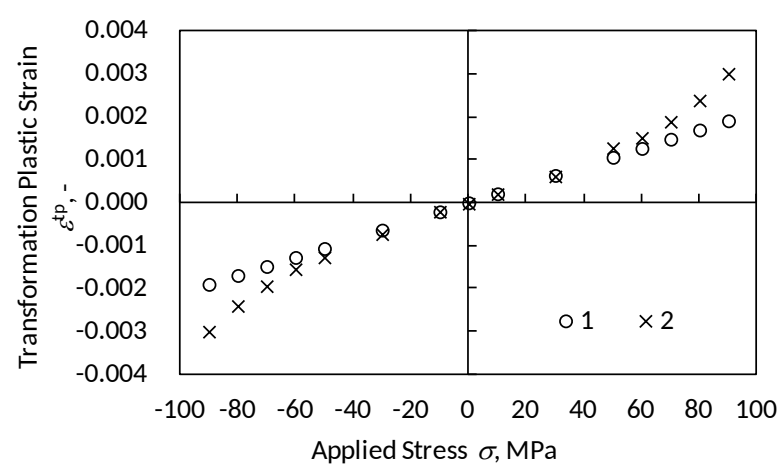

Fig. 22 Difference between analytical solution (circle) and FFT (cross) for $\left\langle\delta \varepsilon_{1}^{e q} / \delta \xi\right\rangle_{V_{1}}$. 


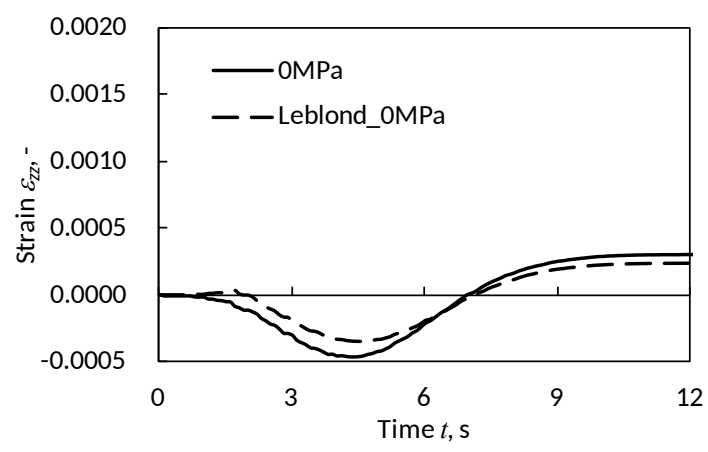

(a) $0 \mathrm{MPa}$

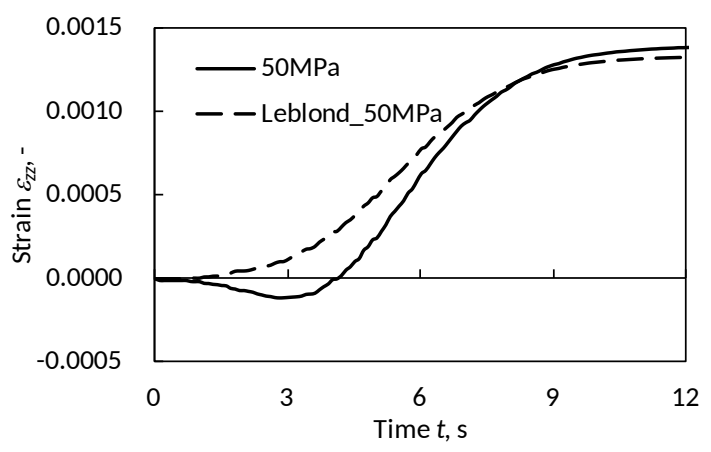

(b) $50 \mathrm{MPa}$

Fig. 23 Relation between FFT and Leblond model results of pre-tension case.

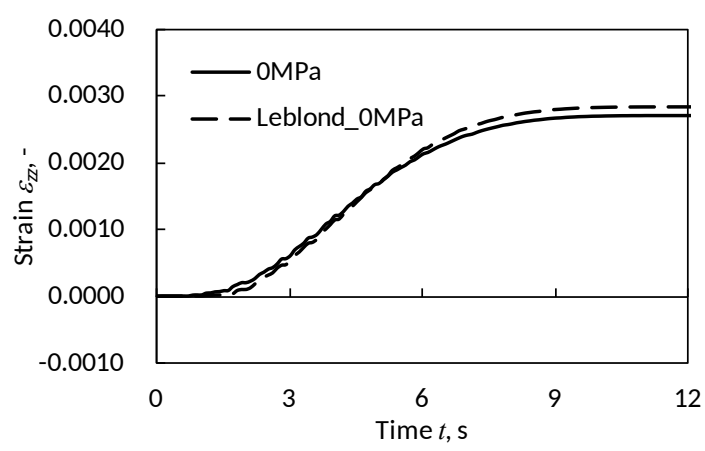

(a) $0 \mathrm{MPa}$

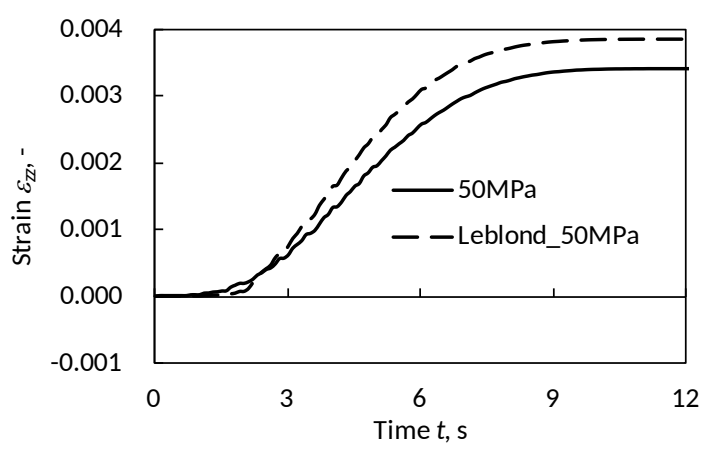

(b) $50 \mathrm{MPa}$

Fig. 24 Relation between FFT and Leblond model results of pre-compression case.

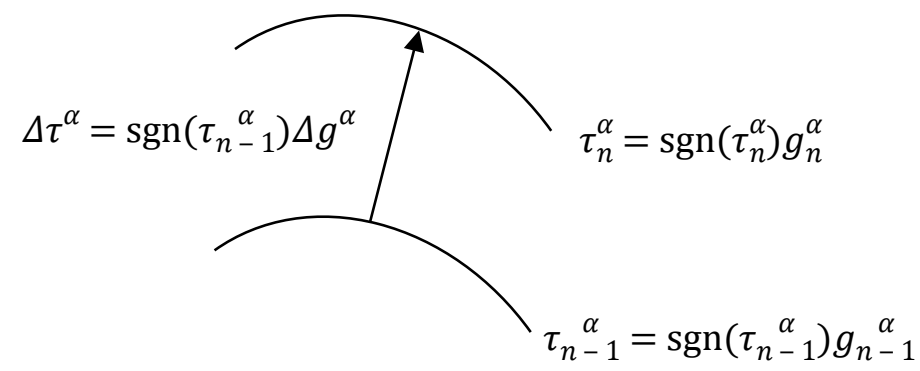

Fig. A-1 Work hardening and resolved stress increment during plastic deformation. 


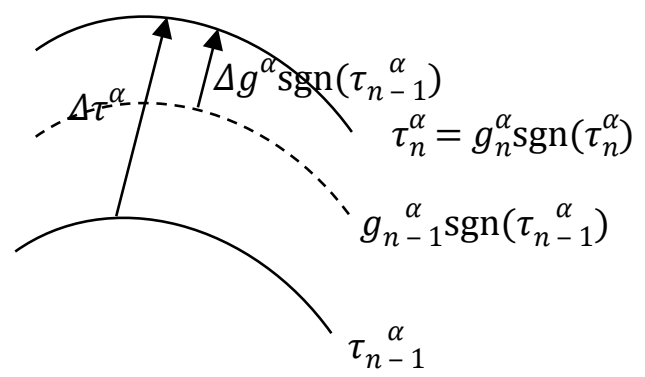

Fig. A-2 Division of trajectory in one step into two paths (elastic and elastoplastic).

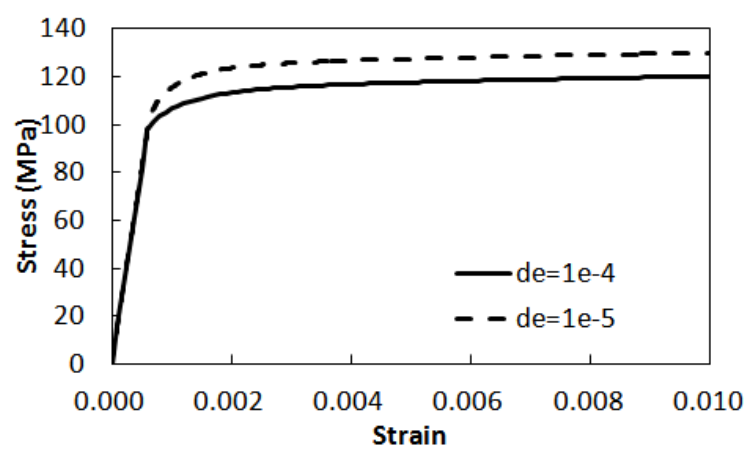

Fig. A-3 Calculated stress-strain curves with different strain increment par step.

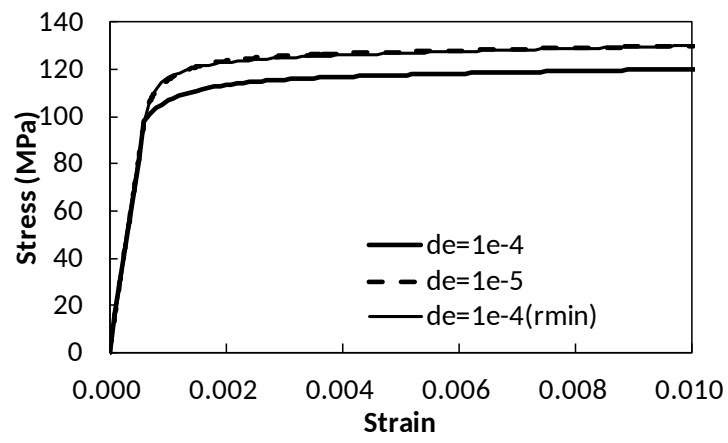

Fig. A-4 Calculated stress-strain curves with the two proposed methods for the determination of the plastic slip increments. 
Table 1 Parameters for crystal plasticity calculation.

\begin{tabular}{|c|c|c|}
\hline & Austenite & Pearlite \\
\hline Bulk modulus $(\mathrm{MPa})$ & 135833 & 150000 \\
\hline Shear modulus $(\mathrm{MPa})$ & 62692 & 69231 \\
\hline$H_{0}(\mathrm{MPa})$ & 10 & 550 \\
\hline$\tau_{0}(\mathrm{MPa})$ & 30 & 100 \\
\hline$\tau_{s}(\mathrm{MPa})$ & 40 & 130 \\
\hline$q$ & 1.0 & 1.0 \\
\hline Number of grains & 100 & 100 \\
\hline
\end{tabular}




\section{FFT-based modelling of transformation plasticity in polycrystalline materials during diffusive phase transformation}

by

Takayuki OTSUKA

Corresponding author

Technical Research \& Development Bureau, Nippon Steel and Sumitomo Metal Corporation, 20-1

Shintomi Futtsu Chiba 293-8511 Japan

otsuka.6gx.takayuki@jp.nssmc.com

Renald BRENNER

Institut Jean le Rond d'Alembert, Université Pierre et Marie Curie, CNRS, UMR7190, 4 place Jussieu 75005 Paris Cedex 5 France

Brigitte BACROIX

Laboratoire des Sciences des Procédés et des Matériaux, Université Paris 13, CNRS, UPR 3407, 99 av. J-B Clément 93430 Villetaneuse France 